\title{
First-Principles Theory of Spatial Dispersion: Dynamical Quadrupoles and Flexoelectricity
}

\author{
Miquel Royo ${ }^{1, *}$ and Massimiliano Stengel ${ }^{1,2, \dagger}$ \\ ${ }^{1}$ Institut de Ciència de Materials de Barcelona (ICMAB-CSIC), Campus UAB, 08193 Bellaterra, Spain \\ ${ }^{2}$ ICREA-Institució Catalana de Recerca i Estudis Avançats, 08010 Barcelona, Spain
}

(Received 21 December 2018; published 12 June 2019)

\begin{abstract}
Density-functional perturbation theory (DFPT) is nowadays the method of choice for the accurate computation of linear and nonlinear-response properties of materials from first principles. A notable advantage of DFPT over alternative approaches is the possibility of treating incommensurate lattice distortions with an arbitrary wave vector $\mathbf{q}$ at essentially the same computational cost as the lattice-periodic case. Here we show that $\mathbf{q}$ can be formally treated as a perturbation parameter and used in conjunction with the established results of perturbation theory (e.g., the " $2 n+1$ " theorem) to perform a long-wave expansion of an arbitrary response function in powers of the wave-vector components. This procedure provides a powerful general framework to access a wide range of spatial dispersion effects that were formerly difficult to calculate by means of first-principles electronic structure methods. In particular, the physical response to the spatial gradient of any external field can now be calculated at negligible cost by using the response functions to uniform perturbations (electric, magnetic, or strain fields) as the only input. We demonstrate our method by calculating the flexoelectric and dynamical quadrupole tensors of selected crystalline insulators and model systems.
\end{abstract}

DOI: 10.1103/PhysRevX.9.021050

\section{INTRODUCTION}

Spatial dispersion refers to the dependence of a material property (e.g., permittivity, conductivity, or phonon frequency) on the wave vector $\mathbf{q}$ at which it is probed, or equivalently, on the gradients of the perturbation and/or the response in real space. Its origin can be traced back to the nonlocality of the microscopic interactions in condensedmatter systems, where the response to an external field (electromagnetic field or atomic displacement) typically occurs over a neighborhood of the point where the field is applied. While in general, such effects are weak and can often be neglected in macroscopic theories, there are several instances where their physical consequences are important, both regarding their fundamental interest and their potential for practical applications. Indeed, with the ongoing interest in nanoscale phenomena, researchers are increasingly facing situations where the relevant scalar, vector, or tensor quantities (e.g., polarization or strain)

\footnotetext{
*Corresponding author. mroyo@icmab.es

Corresponding author. mstengel@icmab.es

Published by the American Physical Society under the terms of the Creative Commons Attribution 4.0 International license. Further distribution of this work must maintain attribution to the author(s) and the published article's title, journal citation, and DOI.
}

Subject Areas: Condensed Matter Physics, Materials Science

display large variations on a very small length scale; this is precisely the regime at which gradient effects can become strong.

Historically, spatial dispersion has been most studied in the context of the optical response. The first-order wavevector dependence of the dielectric susceptibility tensor, for example, is responsible for the natural optical activity $[1,2]$, which is the property of some crystals of rotating the plane of polarization of the transmitted light. Manifestations of spatial dispersion are, however, ubiquitous; they can involve magnetism (the magnetoelectric effect can be regarded as the first-order dispersion of the conductivity [1]) or elastic degrees of freedom as well (the counterpart of optical gyrotropy in phononics is known as acoustical activity [3]). In the latter context, flexoelectricity [4] is arguably the most notable example, as it has been intensely explored both experimentally and theoretically in the past ten years or so $[5,6]$. It describes the polarization response to the gradient of the applied strain, and therefore, it can be understood as the spatial dispersion of the piezoelectric tensor. Being it a universal property of all insulators regardless of crystal symmetry, it provides a tantalizing route to novel electromechanical device concepts [7] and opens the way to many other applications in energy and information technology $[8,9]$.

The long-wavelength regime also occupies a central place in the theory of lattice dynamics in insulators. Indeed, in the $\mathbf{q} \rightarrow 0$ limit, phonons in insulating crystals are associated with macroscopic electric fields that are due 
to the long-range electrostatic interactions between atoms [10]. Identifying and correctly treating such long-range contributions is crucial for a meaningful calculation of the interatomic force constants (IFCs) from first principles $[11,12]$. The long-range IFCs are typically written as electrostatic dipole-dipole terms, which are responsible for the well-known frequency splitting between longitudinal-optical and transverse-optical phonon branches. It is important to note, however, that the dipole-dipole term captures only the leading contribution to the long-range IFCs (dipole-dipole terms decay as $1 / d^{3}$ as a function of the interatomic distance $d$ ). Higher orders $\left(1 / d^{4}\right.$ and faster) are always present but are systematically neglected as their physical consequences are much more subtle.

The next lowest order, for example, involves dipolequadrupole interactions and is responsible for a nonanalytic behavior [13] of the force-constant matrix at $\mathcal{O}\left(q^{1}\right)$; this translates into a $1 / d^{4}$ decay in real space of the corresponding contribution to the IFCs. A quadrupolar response to an atomic displacement requires a brokeninversion symmetry environment to be active and is always (although not exclusively) present in piezoelectric crystals. Interestingly, in his 1972 seminal paper, Martin [14] predicted that the electronic contribution to the piezoelectric tensor can be written as a sublattice sum of the "dynamical quadrupoles," so we expect these couplings to be important in compounds where electromechanical effects are strong. However, viable methods to compute the quadrupole tensor have been lacking to date; this quantity can be regarded as the first-order spatial dispersion of the Born effective charge tensor and is therefore characterized by analogous technical challenges as the calculation of the flexoelectric tensor.

Developing a systematic quantitative theory of such effects would be very desirable to improve their fundamental understanding and support the ongoing experimental efforts. Achieving this goal, however, presents considerable technical difficulties from the point of view of first-principles electronic structure theory due to the inherent breakdown of translational periodicity that a spatial gradient entails. In the case of flexoelectricity [15], for example, several routes have been explored to deal with this issue. Initially, the flexoelectric coefficients were written as realspace moments of the response (either the electronic charge density or the atomic forces) to the displacement of an isolated atom $[16,17]$. Later, the real-space sums were recast as small-q expansions of the response to a monochromatic displacement pattern at a given wave vector $\mathbf{q}[13,18,19]$. Other subtleties were addressed as well, such as the definition and implementation of the current-density response [20], which eventually allowed for the calculation of the bulk flexoelectric tensor within a perturbative framework based on a primitive cell of the crystal [20].

While the strategy of Ref. [20] could be, in principle, generalized to other physical properties, it still presents an important drawback. Several linear-response calculations need to be performed at different $\mathbf{q}$ points in the vicinity of the Brillouin-zone center, and the second-order coefficients (corresponding to the flexoelectric tensor components) are then extracted via a numerical fit. This requirement introduces significant computational overhead (to repeat the same calculations at several values of $\mathbf{q}$ ) and is a potential source of numerical inaccuracies related to the fit. It would be much cheaper from the computational point of view, and convenient from the point of view of the end user, to directly calculate the desired dispersion coefficients as part of the intrinsic linear-response capabilities of the code. To achieve this goal, however, one needs first to establish a general formalism to describe the long-wavelength limit within the context of density-functional perturbation theory (DFPT).

Here we provide a comprehensive solution to the above issues by first rewriting the second-order energy at finite $\mathbf{q}$ as an unconstrained minimization problem of a variational functional of the first-order wave functions. Next, we show that the parametric $\mathbf{q}$ dependence of the second-order energy can be regarded as a small perturbation of the $\mathbf{q}=$ 0 functional; hence, one can apply the standard tools of DFPT to perform an analytic long-wavelength expansion of an arbitrary response property of the crystal in powers of $\mathbf{q}$. Remarkably, this strategy in combination with the " $2 n+1$ " theorem enables us to write explicit formulas for first-order dispersion coefficients that need only the uniform field wave-function response as an input. Thus, one can take advantage of the already implemented linear-response tools to calculate a wide range of new materials properties, such as flexoelectricity and the natural optical activity, at essentially no cost and without the need for explicitly implementing or calculating the wave-function response to a gradient of the external field. Finally, we demonstrate our formalism by implementing the formulas for the clampedion flexoelectric coefficients and the dynamical quadrupole tensor (the higher-order multipolar counterpart of the Born dynamical charge tensor). The flexoelectric coefficients that we calculate for several materials are consistent with previously published results [19,20]. Established by Martin in his seminal paper [14], the relationship between the sublattice sum of the quadrupole moments and the clamped-ion piezoelectric coefficients is numerically verified to a high degree of accuracy. Both quantities converge with respect to the plane-wave cutoff and $\mathbf{k}$-mesh density comparably to "standard" linear-response properties (e.g., the macroscopic dielectric tensor) and can now be obtained in a tiny fraction of the computational burden that was formerly needed.

This work is organized as follows. In Sec. II, we present our method based on the long-wavelength expansion of DFPT and provide general formulas for dispersion properties at the lowest orders in q. In Sec. III, we discuss the finite-q generalization of the electric field response, which 
we use to define and compute the polarization response in the long-wavelength limit. In Secs. IV and V, we demonstrate our long-wave approach by deriving and calculating the dynamical quadrupole and clamped-ion flexoelectric tensors in selected materials and model systems. Finally, in Sec. VI, we present our conclusions and outlook, e.g., regarding future generalizations of our method to other dispersion properties. The Appendixes provide additional analytic support for the formulas reported in the main text.

\section{LONG-WAVE PERTURBATION THEORY}

\section{A. Density-functional perturbation theory}

Here we briefly introduce the basic principles of DFPT, both for completeness and in order to support the formal developments of the later sections. Consider an external perturbation to the electronic ground state, which we describe by assuming a parametric dependence of the Hamiltonian operator on a small parameter $\lambda$,

$$
\hat{H}(\lambda)=\hat{H}^{(0)}+\lambda \hat{H}^{(1)}+\lambda^{2} \hat{H}^{(2)}+\cdots .
$$

The linear response of the wave functions to the perturbation can be recast in terms of a Sternheimer equation,

$$
\hat{Q}\left(H^{(0)}-\epsilon_{m}^{(0)}\right) \hat{Q}\left|\psi_{m}^{(1)}\right\rangle=-\hat{Q} \hat{\mathcal{H}}^{(1)}\left|\psi_{m}^{(0)}\right\rangle,
$$

where $\hat{Q}$ indicates the projector on the unoccupied band manifold, and

$$
\hat{\mathcal{H}}^{(1)}=\hat{H}^{(1)}+\hat{V}^{(1)}
$$

contains, in addition to the external perturbation $\hat{H}^{(1)}$, the self-consistent (SCF) potential response $\hat{V}^{(1)}$ that depends on the first-order electron density as

$$
\begin{aligned}
V^{(1)}(\mathbf{r}) & =\int d^{3} r^{\prime} K_{\mathrm{Hxc}}\left(\mathbf{r}, \mathbf{r}^{\prime}\right) n^{(1)}(\mathbf{r}), \\
n^{(1)}(\mathbf{r}) & =2 \Re \sum_{m}\left\langle\psi_{m}^{(0)} \mid \mathbf{r}\right\rangle\left\langle\mathbf{r} \mid \psi_{m}^{(1)}\right\rangle .
\end{aligned}
$$

$K_{\mathrm{Hxc}}\left(\mathbf{r}, \mathbf{r}^{\prime}\right)$ is the Hartree exchange and correlation (Hxc) kernel, which is defined as the variation of the SCF potential at $\mathbf{r}$ with respect to a charge-density perturbation at $\mathbf{r}^{\prime}$ calculated at the ground-state density $n^{(0)}$,

$$
K_{\mathrm{Hxc}}\left(\mathbf{r}, \mathbf{r}^{\prime}\right)=\left.\frac{\delta V_{\mathrm{Hxc}}(\mathbf{r})}{\delta n\left(\mathbf{r}^{\prime}\right)}\right|_{n^{(0)}}=\left.\frac{\delta^{2} E_{\mathrm{Hxc}}}{\delta n(\mathbf{r}) \delta n\left(\mathbf{r}^{\prime}\right)}\right|_{n^{(0)}} .
$$

The second-order variation of the energy with respect to the perturbation can then be written as

$$
E^{(2)}=\sum_{m}\left\langle\psi_{m}^{(0)}\left|\hat{H}^{(1)}\right| \psi_{m}^{(1)}\right\rangle+\frac{1}{2} \frac{\partial^{2} E}{\partial \lambda^{2}},
$$

where the second term on the right-hand side does not depend on the first-order wave functions,

$$
\frac{1}{2} \frac{\partial^{2} E}{\partial \lambda^{2}}=\sum_{m}\left\langle\psi_{m}^{(0)}\left|\hat{H}^{(2)}\right| \psi_{m}^{(0)}\right\rangle .
$$

One can also recast the linear-response problem as a variational functional of the first-order wave functions [21]

$$
\begin{aligned}
E^{(2)}= & \sum_{m}\left\langle\psi_{m}^{(1)}\left|\left(H^{(0)}-\epsilon_{m}^{(0)}\right)\right| \psi_{m}^{(1)}\right\rangle \\
& +\sum_{m}\left(\left\langle\psi_{m}^{(1)}\left|H^{(1)}\right| \psi_{m}^{(0)}\right\rangle+\left\langle\psi_{m}^{(0)}\left|H^{(1)}\right| \psi_{m}^{(1)}\right\rangle\right) \\
& +\frac{1}{2} \int_{\Omega} \int K_{\mathrm{Hxc}}\left(\mathbf{r}, \mathbf{r}^{\prime}\right) n^{(1)}(\mathbf{r}) n^{(1)}\left(\mathbf{r}^{\prime}\right) d^{3} r d^{3} r^{\prime} \\
& +\frac{1}{2} \frac{\partial^{2} E}{\partial \lambda^{2}}
\end{aligned}
$$

(the double integral of the third line must be taken once over all space and once over the primitive unit cell, whose volume is $\Omega$ ) to be solved within the "parallel-transport gauge" (i.e., under the constraint of orthonormality to the valence manifold $\mathcal{V}$ ),

$$
\left\langle\psi_{j}^{(1)} \mid \psi_{l}^{(0)}\right\rangle=0, \quad j, l \in \mathcal{V} .
$$

Equations (4) and (6) manifestly coincide if the first-order wave functions satisfy the Sternheimer equation (2); however, the latter expression has the virtue of being stationary with respect to variations of $\left|\psi_{m}^{(1)}\right\rangle$, and such a characteristic will have a key importance in the context of this work, as we see shortly.

\section{B. Unconstrained variational formulation}

First, recall the definition of the valence- and conduction-band projectors (we already see the latter in the previous subsection),

$$
\hat{P}=\sum_{n}\left|\psi_{n}^{(0)}\right\rangle\left\langle\psi_{n}^{(0)}\right|, \quad \hat{Q}=1-\hat{P} .
$$

We now use these definitions to write the linear-response problem as an unconstrained variational minimum of the following functional

$$
\begin{aligned}
E^{(2)}= & \sum_{m}\left\langle\psi_{m}^{(1)}\left|\left(\hat{H}^{(0)}+a \hat{P}-\epsilon_{m}^{(0)}\right)\right| \psi_{m}^{(1)}\right\rangle \\
& +\sum_{m}\left\langle\psi_{m}^{(1)}\left|\hat{Q} \hat{H}^{(1)}\right| \psi_{m}^{(0)}\right\rangle+\text { c.c. } \\
& +\frac{1}{2} \int_{\Omega} \int K_{\mathrm{Hxc}}\left(\mathbf{r}, \mathbf{r}^{\prime}\right) n^{(1)}(\mathbf{r}) n^{(1)}\left(\mathbf{r}^{\prime}\right) d^{3} r d^{3} r^{\prime} \\
& +\frac{1}{2} \frac{\partial^{2} E}{\partial \lambda^{2}}
\end{aligned}
$$


Note the explicit introduction of the band projectors in the first and second lines and implicitly in the third line via a redefinition of the first-order electron density,

$$
n^{(1)}(\mathbf{r})=\sum_{m}\left\langle\psi_{m}^{(1)}|\hat{Q}| \mathbf{r}\right\rangle\left\langle\mathbf{r} \mid \psi_{m}^{(0)}\right\rangle+\text { c.c. }
$$

The parameter $a$ is a constant with the dimension of an energy, whose role is to ensure that the matrix element in the first line of Eq. (8), quadratic in the first-order wave functions, is defined positive, and hence, that the functional is stable. To see this point, consider the expectation value of the operator in the round brackets on a valence $(v)$ or conduction $(c)$ state,

$$
\begin{aligned}
& \left\langle\psi_{v}^{(0)}\left|\left(\hat{H}^{(0)}+a \hat{P}-\epsilon_{n}\right)\right| \psi_{v}^{(0)}\right\rangle=\epsilon_{v}+a-\epsilon_{n}, \\
& \left\langle\psi_{c}^{(0)}\left|\left(\hat{H}^{(0)}+a \hat{P}-\epsilon_{n}\right)\right| \psi_{c}^{(0)}\right\rangle=\epsilon_{c}-\epsilon_{n} .
\end{aligned}
$$

As $n$ belongs to the valence band, the matrix element on the conduction state is always positive. Regarding the valence state, for the value $\epsilon_{v}+a-\epsilon_{n}$ to be guaranteed to be positive, it suffices to set $a$ to any positive energy that is larger than the total valence bandwidth.

The insertion of a conduction-band projector $\hat{Q}$ in both the charge density and in the second line of Eq. (8) has the purpose of enforcing the parallel-transport gauge, i.e., that at the variational minimum the solutions $\psi^{(1)}$ be strictly orthogonal to the valence manifold. Indeed, thanks to the projectors $\hat{Q}$, the addition of a small valence component to the trial solution $\psi^{(1)}$ leaves the energy unaltered except for the (quadratic) matrix element in the first line of Eq. (8). The latter term, in turn, always provides a positive contribution to the energy, whose magnitude depends on the parameter $a$. Therefore, $a$ has no influence other than preventing the first-order wave functions from acquiring arbitrarily large components on the valence manifold, which will lead to runaway solutions.

Following these considerations, it is clear that the variational solution of this unconstrained energy functional is unique and corresponds precisely to the constrained minimization procedure described by Gonze [22]. It also leads, by differentiating Eq. (8) with respect to $\left\langle\psi_{m}^{(1)}\right|$, to the form of the Sternheimer equation proposed by Baroni et al. [12],

$$
\left(\hat{H}^{(0)}+a \hat{P}-\epsilon_{m}^{(0)}\right)\left|\psi_{m}^{(1)}\right\rangle=-\hat{Q} \hat{\mathcal{H}}^{(1)}\left|\psi_{m}^{(0)}\right\rangle .
$$

Such a form clearly enforces $\hat{P}\left|\psi_{m}^{(1)}\right\rangle=0$ and reduces to Eq. (2) once the left-hand side is projected on the conduction manifold.

\section{Factorization of the phase}

To appreciate the practical advantages of the unconstrained formulation of the previous subsection, we now apply it to a monochromatic perturbation in a periodic crystal. This can be expressed as a phase times a cellperiodic part,

$$
\hat{H}^{(1)}\left(\mathbf{r}, \mathbf{r}^{\prime}\right)=e^{i \mathbf{q} \cdot \mathbf{r}} \hat{H}_{\mathbf{q}}^{(1)}\left(\mathbf{r}, \mathbf{r}^{\prime}\right)
$$

As customary, we work with the cell-periodic part of the Bloch wave functions by writing

$$
\psi_{m \mathbf{k}}(\mathbf{r})=e^{i \mathbf{k} \cdot \mathbf{r}} u_{m \mathbf{k}}(\mathbf{r})
$$

which allows one to reabsorb the incommensurate phase $e^{i \mathbf{q} \cdot \mathbf{r}}$ by performing appropriate shifts of the states and operators in momentum space.

For the sake of generality, we consider the mixed derivative with respect to two distinct perturbations $\lambda_{1}$ and $\lambda_{2}$, whose physical nature is specified later in this manuscript. (The functional, strictly speaking, is variational only for $\lambda_{1}=\lambda_{2}$; yet, even in the mixed case it preserves the stationary character with respect to small variations in the first-order wave functions.) We implicitly assume that the crystal under study is a time-reversal (TR) symmetric insulator. (A generalization of the formulas to TR-broken materials, while not difficult, will unnecessarily complicate the notation.) The second-order energy can then be written as

$$
\begin{aligned}
E_{\mathbf{q}}^{\lambda_{1}^{*} \lambda_{2}}= & s \int_{\mathrm{BZ}}\left[d^{3} k\right] \sum_{m} E_{m \mathbf{k}, \mathbf{q}}^{\lambda_{i}^{*} \lambda_{2}} \\
& +\frac{1}{2} \int_{\Omega} \int K_{\mathbf{q}}\left(\mathbf{r}, \mathbf{r}^{\prime}\right) n_{\mathbf{q}}^{\lambda_{1} *}(\mathbf{r}) n_{\mathbf{q}}^{\lambda_{2}}\left(\mathbf{r}^{\prime}\right) d^{3} r d^{3} r^{\prime} \\
& +\frac{1}{2} \frac{\partial^{2} E}{\partial \lambda_{1}^{*} \partial \lambda_{2}},
\end{aligned}
$$

where the quantity in the first line is given by

$$
\begin{aligned}
E_{m \mathbf{k}, \mathbf{q}}^{\lambda_{1}^{*} \lambda_{2}}= & \left\langle u_{m \mathbf{k}, \mathbf{q}}^{\lambda_{1}}\left|\left(\hat{H}_{\mathbf{k}+\mathbf{q}}^{(0)}+a \hat{P}_{\mathbf{k}+\mathbf{q}}-\epsilon_{m \mathbf{k}}\right)\right| u_{m \mathbf{k}, \mathbf{q}}^{\lambda_{2}}\right\rangle \\
& +\left\langle u_{m \mathbf{k}, \mathbf{q}}^{\lambda_{1}}\left|\hat{Q}_{\mathbf{k}+\mathbf{q}} \hat{H}_{\mathbf{k}, \mathbf{q}}^{\lambda_{2}}\right| u_{m \mathbf{k}}^{(0)}\right\rangle \\
& +\left\langle u_{m \mathbf{k}}^{(0)}\left|\left(\hat{H}_{\mathbf{k}, \mathbf{q}}^{\lambda_{1}}\right)^{\dagger} \hat{Q}_{\mathbf{k}+\mathbf{q}}\right| u_{m \mathbf{k}, \mathbf{q}}^{\lambda_{2}}\right\rangle,
\end{aligned}
$$

$s=2$ is the spin multiplicity, and we use the following shorthand notation for the Brillouin-zone averages,

$$
\int_{\mathrm{BZ}}\left[d^{3} k\right]=\frac{\Omega}{(2 \pi)^{3}} \int_{\mathrm{BZ}} d^{3} k .
$$

The last (third) line in Eq. (12) is, as usual, the nonvariational contribution to the second-order energy, while the second line contains the self-consistent energy that depends quadratically on the first-order electron densities [23] 


$$
n_{\mathbf{q}}^{\lambda}(\mathbf{r})=2 s \int_{\mathrm{BZ}}\left[d^{3} k\right] \sum_{m}\left\langle u_{m \mathbf{k}}^{(0)} \mid \mathbf{r}\right\rangle\left\langle\mathbf{r}\left|\hat{Q}_{\mathbf{k}+\mathbf{q}}\right| u_{m \mathbf{k}, \mathbf{q}}^{\lambda}\right\rangle .
$$

Note that we introduce new symbols for the phasecorrected Hxc kernel [we specialize to the local density approximation (LDA)]

$$
K_{\mathbf{q}}\left(\mathbf{r}, \mathbf{r}^{\prime}\right)=K_{\mathrm{Hxc}}\left(\mathbf{r}, \mathbf{r}^{\prime}\right) e^{i \mathbf{q} \cdot\left(\mathbf{r}^{\prime}-\mathbf{r}\right)}
$$

the operators in momentum space

$$
\hat{O}_{\mathbf{k}}=e^{-i \mathbf{k} \cdot \mathbf{r}} \hat{O} e^{i \mathbf{k} \cdot \mathbf{r}^{\prime}}
$$

and the cell-periodic part of the charge-density response

$$
n_{\mathbf{q}}^{\lambda}(\mathbf{r})=e^{-i \mathbf{q} \cdot \mathbf{r}} n^{\lambda}(\mathbf{r}) .
$$

From these formulas, one can now appreciate the most remarkable property of the unconstrained functional: Unlike the original version, where the orthonormality constraint is taken by calculating the scalar products with ground-state valence orbitals at $\mathbf{k}+\mathbf{q}$, the present version is written in a manifestly gauge-invariant form; i.e., only the operators explicitly depend on q. (The first-order wave functions should be regarded as "trial" solutions, which means that their $\mathbf{q}$ dependence is implicit: It is a consequence of the stationary condition that is imposed on the functional at each q.) This feature is a key advantage when developing a perturbative theory in $\mathbf{q}$, as the derivatives of the operators in momentum space are well-defined mathematical objects and do not suffer from the phase indeterminacy of the Bloch states.

\section{D. $2 n+1$ theorem}

At this point, we can treat $E_{\mathbf{q}}^{\lambda_{1}^{*} \lambda_{2}}$ as a new functional of $\left|u_{m \mathbf{k}, \mathbf{q}}^{\lambda_{1,2}}\right\rangle$, which depends parametrically on $\mathbf{q}$. We can then take advantage of the established mathematical tools of perturbation theory to expand $E_{\mathbf{q}}^{\lambda_{1}^{*} \lambda_{2}}$ in powers of $\mathbf{q}$ around $\mathbf{q}=0$, which has the physical interpretation of a long-wave expansion. This procedure can be pushed, in principle, to any order in q. In particular, in virtue of the $2 n+1$ theorem [24], the knowledge of the $\mathbf{q}$ derivatives of the wave functions up to order $n$ is sufficient to calculate response properties up to $\mathcal{O}\left(q^{2 n+1}\right)$. As we see in the following, this result is especially useful at the lowest orders: The computational tools to calculate the $n=0$ (and, sometimes, $n=1$ ) response functions are already available in many public first-principles packages, which implies that many response properties can be, in principle, extracted without even implementing a new response function in the code. (In the following, we illustrate this strategy at a formal level, without specifying the physical nature of the perturbations; practical examples are provided in Secs. IV and V.)
At first order in $\mathbf{q}$, the $2 n+1$ theorem reduces to the Hellmann-Feynman theorem and can be summarized as follows:

$$
E_{\gamma}^{\lambda_{\gamma}^{*} \lambda_{2}}=\left.\frac{d E_{\mathbf{q}}^{\lambda_{1}^{*} \lambda_{2}}}{d q_{\gamma}}\right|_{\mathbf{q}=0}=\left.\frac{\partial E_{\mathbf{q}}^{\lambda_{1}^{*} \lambda_{2}}}{\partial q_{\gamma}}\right|_{\mathbf{q}=0},
$$

which states that the $\mathbf{q}$ gradients of the response functions $\left|u_{m \mathbf{k}, \mathbf{q}}^{\lambda}\right\rangle$ are not needed to access the $\mathbf{q}$ gradient of the stationary second-order functional. (We specialize our formulas to a neighborhood of $\mathbf{q}=0$, as such a limit is directly relevant for the macroscopic response properties of the crystal.) In particular, we have

$$
\begin{aligned}
E_{\gamma}^{\lambda_{1}^{*} \lambda_{2}}= & s \int_{\mathrm{BZ}}\left[d^{3} k\right] \sum_{m} E_{m \mathbf{k}, \gamma}^{\lambda_{1}^{*} \lambda_{2}} \\
& +\frac{1}{2} \int_{\Omega} \int K_{\gamma}\left(\mathbf{r}, \mathbf{r}^{\prime}\right) n^{\lambda_{1} *}(\mathbf{r}) n^{\lambda_{2}}\left(\mathbf{r}^{\prime}\right) d^{3} r d^{3} r^{\prime} \\
& +\left.\frac{1}{2} \frac{\partial}{\partial q_{\gamma}}\left(\frac{\partial^{2} E}{\partial \lambda_{1}^{*} \partial \lambda_{2}}\right)\right|_{\mathbf{q}=0},
\end{aligned}
$$

where we use shorthand notation for the $\mathbf{q}$ derivative of the Hartree and exchange-correlation kernel,

$$
K_{\gamma}\left(\mathbf{r}, \mathbf{r}^{\prime}\right)=\left.\frac{\partial K_{\mathbf{q}}\left(\mathbf{r}, \mathbf{r}^{\prime}\right)}{\partial q_{\gamma}}\right|_{\mathbf{q}=0},
$$

and the band-resolved contribution reads as

$$
\begin{aligned}
E_{m \mathbf{k}, \gamma}^{\lambda_{1}^{*} \lambda_{2}}= & \left\langle u_{m \mathbf{k}}^{\lambda_{1}}\left|\partial_{\gamma} \hat{H}_{\mathbf{k}}^{(0)}\right| u_{m \mathbf{k}}^{\lambda_{2}}\right\rangle \\
& +\left\langle u_{m \mathbf{k}}^{\lambda_{1}}\left|\partial_{\gamma} \hat{Q}_{\mathbf{k}} \hat{\mathcal{H}}_{\mathbf{k}}^{\lambda_{2}}\right| u_{m \mathbf{k}}^{(0)}\right\rangle+\left\langle u_{m \mathbf{k}}^{(0)}\left|\left(\hat{\mathcal{H}}_{\mathbf{k}}^{\lambda_{1}}\right)^{\dagger} \partial_{\gamma} \hat{Q}_{\mathbf{k}}\right| u_{m \mathbf{k}}^{\lambda_{2}}\right\rangle \\
& +\left\langle u_{m \mathbf{k}}^{\lambda_{1}}\left|\hat{H}_{\mathbf{k}, \gamma}^{\lambda_{2}}\right| u_{m \mathbf{k}}^{(0)}\right\rangle+\left\langle u_{m \mathbf{k}}^{(0)}\left|\left(\hat{H}_{\mathbf{k}, \gamma}^{\lambda_{1}}\right)^{\dagger}\right| u_{m \mathbf{k}}^{\lambda_{2}}\right\rangle .
\end{aligned}
$$

Here we introduce new symbols for the $\mathbf{q}$ derivatives of the external perturbation,

$$
\hat{H}_{\mathbf{k}, \gamma}^{\lambda}=\left.\frac{\partial \hat{H}_{\mathbf{k}, \mathbf{q}}^{\lambda}}{\partial q_{\gamma}}\right|_{\mathbf{q}=0},
$$

and for the $\mathbf{k}$ derivatives of the ground-state operators (Hamiltonian or band projectors), e.g.,

$$
\partial_{\gamma} \hat{H}_{\mathbf{k}}^{(0)}=\left.\frac{\partial \hat{H}_{\mathbf{k}+\mathbf{q}}^{(0)}}{\partial q_{\gamma}}\right|_{\mathbf{q}=0} .
$$

Also, we remove the $\mathbf{q}$ subscript from those quantities (either first-order wave functions, densities, or perturbing operators) that are intended to be calculated at $\mathbf{q}=0$, e.g., $\left|u_{m \mathbf{k}}^{\lambda}\right\rangle=\left|u_{m \mathbf{k}, \mathbf{q}=0}^{\lambda}\right\rangle$.

Note that we use the symbol $\hat{\mathcal{H}}$ introduced in Eq. (3) in the second line of Eq. (17) to indicate that the self-consistent (SCF) Hartree and exchange-correlation 
potential must be included in the first-order Hamiltonian at $\mathbf{q}=0$. The SCF part of $\hat{\mathcal{H}}_{\mathbf{k}}^{\lambda}$ comes from the Hartree and exchange-correlation term in Eq. (12) via the partial derivative of the first-order density with respect to $q_{\gamma}$,

$$
\frac{\partial n^{\lambda}(\mathbf{r})}{\partial q_{\gamma}}=2 s \int_{\mathrm{BZ}}\left[d^{3} k\right] \sum_{m}\left\langle u_{m \mathbf{k}}^{(0)} \mid \mathbf{r}\right\rangle\left\langle\mathbf{r}\left|\partial_{\gamma} \hat{Q}_{\mathbf{k}}\right| u_{m \mathbf{k}}^{\lambda}\right\rangle .
$$

Crucially, the SCF potential needs to be explicitly calculated only at the level of the $\mathcal{O}\left(q^{0}\right)$ perturbation; the $\mathbf{q}$ gradient of the perturbation, in the third line, concerns only the external potential part $\hat{H}$. (This result is, again, a consequence of the $2 n+1$ theorem.) Note also that $\partial_{\gamma} \hat{P}_{\mathbf{k}}$ has only cross-gap matrix elements; thus, it does not contribute to the first line of Eq. (17), and we can omit $\hat{Q}_{\mathbf{k}}$ from the matrix elements in the third line, as it always appears next to a conduction-band state.

The above formulas enable the calculation of the " $d / d q_{\gamma}$ " response with a computational workload that is comparable to the uniform $(\mathbf{q}=0)$ case. Indeed, only the $\mathbf{q}=0$ first-order wave functions are needed as ingredients; the additional burden consists of the implementation of the new operators that appear in Eqs. (16) and (17), but once this is done, the evaluation of the corresponding matrix elements proceeds at essentially no cost. Most of these "new" operators are, in fact, well known in the context of band theory and are standard in most DFPT implementations (e.g., the velocity operator $\partial_{\gamma} \hat{H}_{\mathbf{k}}^{(0)}$ or the derivatives of the band projectors). For example, the second line of Eq. (17) might look unusual at first sight, but it can be made more explicit by observing that $\partial_{\gamma} \hat{Q}_{\mathbf{k}}=-\partial_{\gamma} \hat{P}_{\mathbf{k}}$ and that

$$
\partial_{\gamma} \hat{P}_{\mathbf{k}}=\sum_{n}\left(\left|u_{n \mathbf{k}}^{(0)}\right\rangle\left\langle\tilde{\partial}_{\gamma} u_{n \mathbf{k}}^{(0)}|+| \tilde{\partial}_{\gamma} u_{n \mathbf{k}}^{(0)}\right\rangle\left\langle u_{n \mathbf{k}}^{(0)}\right|\right),
$$

where $\left|\tilde{\partial}_{\gamma} u_{n \mathbf{k}}^{(0)}\right\rangle$ are the "covariant derivatives" of the ground-state wave functions (also known as $d / d k_{\gamma}$ response functions) and are orthogonal to the valence manifold. Then, one immediately obtains

$$
\left\langle u_{m \mathbf{k}}^{\lambda_{1}}\left|\partial_{\gamma} \hat{Q}_{\mathbf{k}} \hat{\mathcal{H}}_{\mathbf{k}}^{\lambda_{2}}\right| u_{m \mathbf{k}}^{(0)}\right\rangle=-\sum_{n}\left\langle u_{m \mathbf{k}}^{\lambda_{1}} \mid \tilde{\partial}_{\gamma} u_{n \mathbf{k}}^{(0)}\right\rangle\left\langle u_{n \mathbf{k}}^{(0)}\left|\hat{\mathcal{H}}_{\mathbf{k}}^{\lambda_{2}}\right| u_{m \mathbf{k}}^{(0)}\right\rangle
$$

which is now a rather familiar expression in the context of DFPT.

The truly new pieces in Eqs. (16) and (17) are the q derivatives of the monochromatic perturbation and the $\mathbf{q}$ derivative of the SCF kernel. We defer the discussion of the former term, which depends on the specific perturbation, to Secs. IV and V. The latter quantity is particularly simple to evaluate in the framework of the LDA, where the XC kernel is independent of q. (Adapting the formalism to other XC functionals, e.g., in the framework of the generalizedgradient approximation is, in any case, straightforward: It requires calculating only the analytic $\mathbf{q}$ derivative of the exchange-correlation kernel, which is well behaved in the long-wavelength limit [25]) As we are left only with electrostatic effects, it is most convenient to work in reciprocal space, where the Coulomb (Hartree) kernel is local,

$$
K_{H, \mathbf{q}}\left(\mathbf{G}, \mathbf{G}^{\prime}\right)=4 \pi \frac{\delta_{\mathbf{G G}^{\prime}}}{|\mathbf{G}+\mathbf{q}|^{2}}
$$

( $\mathbf{G}$ and $\mathbf{G}^{\prime}$ stand for reciprocal-lattice vectors, and $\delta$ is a Kronecker symbol.) The $\mathbf{q}$ gradient (at $\mathbf{q}=0$ ) of the above expression is easily computed,

$$
K_{\gamma}\left(\mathbf{G}, \mathbf{G}^{\prime}\right)=-8 \pi G_{\gamma} \frac{\delta_{\mathbf{G G}^{\prime}}}{G^{4}} .
$$

The $\mathbf{G}=0$ term must be, of course, excluded; this corresponds to adopting short-circuit electrical boundary conditions, which is the correct choice for computing materials properties that have a tensorial nature. (A formal justification of this point was provided in Ref. [22] for the uniform electric field problem and in Ref. [13] for the flexoelectric tensor.)

\section{E. Higher orders}

As we said, the $2 n+1$ theorem, in principle, provides access to the long-wave expansion terms of a given crystal response to any order in $\mathbf{q}$. In general, the analytic formulas for higher orders in $\mathbf{q}$ can become rather cumbersome to derive, as they involve a larger number of terms; plus, they typically require additional response functions to be implemented and calculated. There is, however, an important exception to this statement that is worth discussing, as it is central to the topics that we present in the later sections. Indeed, there are some notable cases where a perturbation produces a vanishing response at $\mathbf{q}=0$, and the interesting physics occurs only at first order in $\mathbf{q}$. A classic example is that of a scalar potential perturbation: At $\mathbf{q}=0$, the perturbation is a rigid shift of the potential reference, which has obviously no effect on the electronic structure; at first order in $\mathbf{q}$, one obtains the response to a uniform electric field [22]. In such cases, the formula for the $\mathcal{O}\left(q^{2}\right)$ response simplifies considerably and, in fact, is only marginally more complicated than the first-order formulas, Eqs. (16) and (17).

To be more specific, consider the following mixed derivative,

$$
E_{\gamma \delta}^{\lambda_{1}^{*} \lambda_{2}}=\left.\frac{d^{2} E_{\mathbf{q}}^{\lambda_{1}^{*} \lambda_{2}}}{d q_{\gamma} d q_{\delta}}\right|_{\mathbf{q}=0},
$$


and assume that $\left|u_{m \mathbf{k}, \mathbf{q}=0}^{\lambda_{2}}\right\rangle=0$. Consistent with the above notation, we indicate the response function to a gradient of the perturbation $\lambda_{2}$ as

$$
\left.\left|u_{m \mathbf{k}, \delta}^{\lambda_{2}}\right\rangle=\left|\frac{\partial u_{m \mathbf{k}, \mathbf{q}}^{\lambda_{2}}}{\partial q_{\delta}}\right|_{\mathbf{q}=0}\right\rangle .
$$

Then we have

$$
E_{\gamma \delta}^{\lambda_{1}^{*} \lambda_{2}}=\tilde{E}_{\gamma \delta}^{\lambda_{1}^{*} \lambda_{2}}+\tilde{E}_{\delta \gamma}^{\lambda_{1}^{*} \lambda_{2}}
$$

where the tilded (unsymmetrized) quantities read as

$$
\begin{aligned}
\tilde{E}_{\gamma \delta}^{\lambda_{1}^{*} \lambda_{2}}= & s \int_{\mathrm{BZ}}\left[d^{3} k\right] \sum_{m} \tilde{E}_{m \mathbf{k}, \gamma \delta}^{\lambda_{1}^{*} \lambda_{2}} \\
& +\frac{1}{2} \iint K_{\gamma}\left(\mathbf{r}, \mathbf{r}^{\prime}\right) n^{\lambda_{1} *}(\mathbf{r}) n_{\delta}^{\lambda_{2}}\left(\mathbf{r}^{\prime}\right) d^{3} r d^{3} r^{\prime} \\
& +\left.\frac{1}{4} \frac{\partial^{2}}{\partial q_{\gamma} \partial q_{\delta}}\left(\frac{\partial^{2} E}{\partial \lambda_{1}^{*} \partial \lambda_{2}}\right)\right|_{\mathbf{q}=0}
\end{aligned}
$$

with

$$
\begin{aligned}
\tilde{E}_{m \mathbf{k}, \gamma \delta}^{\lambda_{1}^{\lambda} \lambda_{2}}= & \left\langle u_{m \mathbf{k}}^{\lambda_{1}}\left|\partial_{\gamma} \hat{H}_{\mathbf{k}}^{(0)}\right| u_{m \mathbf{k}, \delta}^{\lambda_{2}}\right\rangle \\
& +\frac{1}{2}\left\langle u_{m \mathbf{k}}^{\lambda_{1}}\left|\partial_{\gamma \delta} \hat{Q}_{\mathbf{k}} \hat{\mathcal{H}}_{\mathbf{k}}^{\lambda_{2}}\right| u_{m \mathbf{k}}^{(0)}\right\rangle+\left\langle u_{m \mathbf{k}}^{\lambda_{1}}\left|\partial_{\gamma} \hat{Q}_{\mathbf{k}} \hat{\mathcal{H}}_{\mathbf{k}, \delta}^{\lambda_{2}}\right| u_{m \mathbf{k}}^{(0)}\right\rangle \\
& +\left\langle u_{m \mathbf{k}}^{(0)}\left|\left(\hat{\mathcal{H}}_{\mathbf{k}}^{\lambda_{1}}\right)^{\dagger} \partial_{\gamma} \hat{Q}_{\mathbf{k}}\right| u_{m \mathbf{k}, \delta}^{\lambda_{2}}\right\rangle+\frac{1}{2}\left\langle u_{m \mathbf{k}}^{\lambda_{1}}\left|\hat{H}_{\mathbf{k}, \gamma \delta}^{\lambda_{2}}\right| u_{m \mathbf{k}}^{(0)}\right\rangle \\
& +\left\langle u_{m \mathbf{k}}^{(0)}\left|\left(\hat{H}_{\mathbf{k}, \gamma}^{\lambda_{1}}\right)^{\dagger}\right| u_{m \mathbf{k}, \delta}^{\lambda_{2}}\right\rangle
\end{aligned}
$$

and

$$
n_{\delta}^{\lambda_{2}}(\mathbf{r})=2 s \int_{\mathrm{BZ}}\left[d^{3} k\right] \sum_{m}\left\langle u_{m \mathbf{k}}^{(0)} \mid \mathbf{r}\right\rangle\left\langle\mathbf{r} \mid u_{m \mathbf{k}, \delta}^{\lambda_{2}}\right\rangle .
$$

(Again, we can drop the conduction-band projector as $\left|u_{m \mathbf{k}, \delta}^{\lambda_{2}}\right\rangle$ belongs to the conduction band by construction.) The resulting formulas for the second-order energy are essentially identical to those derived in Sec. II D for the first order in $\mathbf{q}$, with three main differences: (i) the result needs now to be symmetrized with respect to $\gamma$ and $\delta$; (ii) every occurrence of the response functions and perturbing operators that depend on $\lambda_{2}$ need to be replaced with their next higher-order gradient in $\mathbf{q}$; (iii) there is a new term in Eq. (25) containing the second $\mathbf{k}$ gradient of the band projector $\partial_{\gamma \delta} \hat{Q}_{\mathbf{k}}$. The latter operator is multiplied by $\hat{\mathcal{H}}_{\mathbf{k}}^{\lambda_{2}}$, which we include to account for cases where the perturbation $\lambda_{2}$, while yielding a vanishing response at $\mathbf{q}=0$, may not vanish therein.

Similar considerations can be used in order to push the expansion to $\mathcal{O}\left(q^{3}\right)$ whenever both perturbations $\lambda_{1}$ and $\lambda_{2}$ produce a vanishing response at $\mathbf{q}=0$.

\section{TREATMENT OF THE POLARIZATION RESPONSE}

Many materials properties (including the flexoelectric tensor that we discuss in Sec. V) involve, in one way or another, the polarization response to an external perturbation. Correctly treating the long-wavelength limit of the electrical polarization is far from trivial in the framework of density-functional perturbation theory. In the presence of a spatial modulation, the standard formulas (e.g., based on the Berry-phase approach) are not applicable, since they are specialized to the macroscopic response at the Brillouinzone center. To work around this issue, in Ref. [20] the polarization $(\mathbf{P})$ response to some monochromatic external field $\lambda^{\mathbf{q}}$ was expressed as the current-density $(\mathbf{J})$ response to the time derivative of the field,

$$
\frac{d \mathbf{P}^{\mathbf{q}}}{d \lambda^{\mathbf{q}}}=\frac{d \mathbf{J}^{\mathbf{q}}}{d \dot{\lambda}^{\mathbf{q}}} .
$$

In a quantum-mechanical context, this can be expressed $[20,26]$ via the following formula,

$$
\frac{d P_{\alpha}^{\mathbf{q}}}{d \lambda^{\mathbf{q}}}=\frac{2 s}{\Omega} \int_{\mathrm{BZ}}\left[d^{3} k\right] \sum_{m}\left\langle u_{m \mathbf{k}}^{(0)}\left|\hat{J}_{\alpha \mathbf{k}, \mathbf{q}}\right| \delta u_{m \mathbf{k}, \mathbf{q}}^{\lambda}\right\rangle,
$$

where $\hat{J}_{\alpha \mathbf{k}, \mathbf{q}}$ is the current-density operator at a given value of $\mathbf{q},\left|\delta u_{m \mathbf{k}, \mathbf{q}}^{\lambda}\right\rangle$ describes the adiabatic wave-function response [20] to the perturbation velocity in the limit of $\dot{\lambda}^{q} \rightarrow 0$, and the index $m$ runs over the occupied manifold. In other words, if we modulate the perturbation in time with a dynamical phase $e^{-i \omega t},\left|\delta \psi_{i}^{\mathbf{q}}\right\rangle$ is related to the first-order term in the low-frequency expansion of the wave-function response.

Unfortunately, Eq. (28) is not directly useful to our scopes, as it is not explicitly written as a second derivative of the total energy. To circumvent this issue, we use the known [27] thermodynamic relationship $\mathbf{P}=-\partial E / \partial \mathcal{E}$ to rewrite $\mathbf{P}$ as a mixed derivative with respect to the electric field $\mathcal{E}$ and the external perturbation $\lambda$,

$$
\frac{d P_{\alpha}^{\mathbf{q}}}{d \lambda^{\mathbf{q}}}=-\frac{d^{2} E}{d \mathcal{E}_{\alpha}^{-\mathbf{q}} d \lambda^{\mathbf{q}}} .
$$

This strategy recovers the established DFPT formulas [11] for the polarization response in the $\mathbf{q}=0$ case. [For instance, if $\lambda$ is an atomic displacement, Eq. (29) reduces to the standard linear-response expression for the Born effective charge tensor.] It presents, however, a new complication in that we need to generalize the electric field perturbation to finite values of q. To do that, we express the $\mathcal{E}$-field perturbation as the time derivative of the A-field perturbation, again by means of adiabatic perturbation theory. As we see shortly, this allows us to write the polarization response in a variational form, and therefore 
apply the formalism that we develop in the previous sections to perform its long-wave expansion.

In the following subsections, we first discuss the response to a monochromatic vector potential at finite $\mathbf{q}$, which is the fundamental building block of our approach. The electric field response is then defined as the frequency derivative of the vector potential response via a first-order expansion in the frequency. Finally, we discuss the simpler case of the scalar potential perturbation and show that, at first order in $\mathbf{q}$, it correctly recovers the electric field response (as defined via adiabatic perturbation theory) at $\mathbf{q}=0$.

\section{A. Vector potential}

The coupling of a generic Hamiltonian to a modulated $\mathbf{A}$ field is expressed, in the linear regime, via the currentdensity operator [20]. We report its derivation, following the guidelines of Ref. [20], in Appendix A; hereafter, we discuss the response to such a perturbation with special attention to the lowest orders in $\mathbf{q}$.

The wave-function response can be written in terms of the following Sternheimer equation

$$
\left(\hat{H}_{\mathbf{k}+\mathbf{q}}^{(0)}+a \hat{P}_{\mathbf{k}+\mathbf{q}}-\epsilon_{m \mathbf{k}}\right)\left|u_{m \mathbf{k}, \mathbf{q}}^{A_{\alpha}}\right\rangle=-\hat{Q}_{\mathbf{k}+\mathbf{q}} \hat{H}_{\mathbf{k}, \mathbf{q}}^{A_{\alpha}}\left|u_{m \mathbf{k}}^{(0)}\right\rangle,
$$

where $\hat{H}_{\mathbf{k}, \mathbf{q}}^{A_{\alpha}}$ is the first-order variation of the Hamiltonian in the presence of a modulated $\mathbf{A}$ field. (Note the absence of the SCF potential contribution, as a static vector potential field leaves the charge density of the crystal unaltered in the linear regime by time-reversal symmetry.) In the context of this work, we need only the zeroth and first orders in the $\mathbf{q}$ expansion of $\left|u_{m \mathbf{k}, \mathbf{q}}^{A_{\alpha}}\right\rangle$. Regarding the $\mathbf{q}=0$ limit, it is easy to show that (since we are dealing with electrons, we assume $\mathcal{Q}=-1$ henceforth)

$$
\left|u_{m \mathbf{k}, \mathbf{q}=0}^{A_{\alpha}}\right\rangle=\partial_{\alpha} \hat{P}_{\mathbf{k}}\left|u_{m \mathbf{k}}^{(0)}\right\rangle=\left|\tilde{\partial}_{\alpha} u_{m \mathbf{k}}^{(0)}\right\rangle
$$

where the " $\partial$ " sign is a shortcut for the gradient in $\mathbf{k}$ space, and the tilde indicates the covariant derivation in the language of band theory. Regarding the first order in $\mathbf{q}$, we report here the final result (we report a detailed derivation in Appendix A)

$$
\begin{aligned}
\left|u_{m \mathbf{k}, \gamma}^{A_{\beta}}\right\rangle= & \left|\frac{\partial u_{m \mathbf{k}, \mathbf{q}=0}^{A_{\beta}}}{\partial q_{\gamma}}\right\rangle=\frac{1}{2}\left(\partial_{\beta \gamma}^{2} \hat{P}_{\mathbf{k}}\left|u_{n \mathbf{k}}^{(0)}\right\rangle\right. \\
& \left.-\left[\partial_{\gamma} \hat{P}_{\mathbf{k}}, \partial_{\beta} \hat{P}_{\mathbf{k}}\right]\left|u_{n \mathbf{k}}^{(0)}\right\rangle+\left|u_{n \mathbf{k}, \beta \gamma}^{\mathrm{CG}}\right\rangle\right) .
\end{aligned}
$$

The first term on the right-hand side is symmetric in $\beta \gamma$; the second and the third terms are both antisymmetric and describe the response to a uniform magnetic field $\mathbf{B}$. In particular, the second contribution has only valence-band components and is related to the Berry curvature; the third is a cross-gap (CG) contribution that obeys the following Sternheimer equation,

$$
\begin{aligned}
\left(\hat{H}_{\mathbf{k}}+a \hat{P}_{\mathbf{k}}-\epsilon_{n \mathbf{k}}\right)\left|u_{n \mathbf{k}, \beta \gamma}^{\mathrm{CG}}\right\rangle= & -\hat{Q}_{\mathbf{k}}\left(\left\{\partial_{\gamma} \hat{H}_{\mathbf{k}}, \partial_{\alpha} \hat{P}_{\mathbf{k}}\right\}\right. \\
& \left.-\left\{\partial_{\alpha} \hat{H}_{\mathbf{k}}, \partial_{\gamma} \hat{P}_{\mathbf{k}}\right\}\right)\left|u_{n \mathbf{k}}^{(0)}\right\rangle .
\end{aligned}
$$

This corresponds precisely to the linear response of the wave functions to a uniform $\mathbf{B}$ field as derived in Ref. [28].

\section{B. Electric field}

The standard treatment of the electric field perturbation is based on the long-wavelength limit of a scalar potential perturbation [22]. Such an approach, which we discuss in Sec. III C, is appealing for its simplicity; however, when pushed to higher orders in $\mathbf{q}$, it has the disadvantage of limiting the scope of the theory to the longitudinal components of many dispersion-related tensors. (The transverse components of the flexoelectric tensor, for example, require a current-density response theory [20], while the scalar potential is only sensitive to the charge-density response.)

Instead, here we work in an electromagnetic gauge where the scalar potential vanishes, and the electric field is provided by a vector potential that is slowly varying over time $\mathcal{E}=-\partial_{t} \mathbf{A}$. To achieve this goal, we need to establish a time-dependent framework, where the external perturbation (in this case, the vector potential that we discuss in the previous subsection) is applied dynamically.

The adequate formalism to attack this problem is provided by first-order adiabatic perturbation theory, which relates the adiabatic wave functions $|\delta n\rangle$ to the static response functions $\left|\partial_{\lambda} n\right\rangle$ via a Sternheimer equation,

$$
\left(\hat{H}+a \hat{P}-\epsilon_{n}\right)|\delta n\rangle=i\left|\partial_{\lambda} n\right\rangle .
$$

Here, $\left|\partial_{\lambda} n\right\rangle$ and $|\delta n\rangle$ describe the first-order response to $\lambda$ and $\dot{\lambda}$, respectively. In the context of the electric field response, this translates into

$$
\begin{aligned}
& \left(\hat{H}_{\mathbf{k}+\mathbf{q}}^{(0)}+a \hat{P}_{\mathbf{k}+\mathbf{q}}-\epsilon_{m \mathbf{k}}\right)\left|u_{m \mathbf{k}, \mathbf{q}}^{\mathcal{E}_{\alpha}}\right\rangle \\
& \quad=-i\left|u_{m \mathbf{k}, \mathbf{q}}^{A_{\alpha}}\right\rangle-\hat{Q}_{\mathbf{k}+\mathbf{q}} V_{\mathbf{q}}^{\mathcal{E}_{\alpha}}\left|u_{n \mathbf{k}}^{(0)}\right\rangle,
\end{aligned}
$$

where we incorporate charge self-consistency via the usual SCF potential contribution $V_{\mathbf{q}}^{\mathcal{E}_{\alpha}}$. Remarkably, the A-field response functions now play the role of an external perturbation in the context of the $\mathbf{E}$-field response,

$$
\hat{Q}_{\mathbf{k}+\mathbf{q}} \hat{H}_{\mathbf{k}, \mathbf{q}}^{\mathcal{E}_{\alpha}}\left|u_{m \mathbf{k}}^{(0)}\right\rangle \rightarrow\left|i u_{m \mathbf{k}, \mathbf{q}}^{A_{\alpha}}\right\rangle .
$$

This allows us to write the mixed derivative with respect to an electric field and a second perturbation $\lambda$ as the following stationary functional of $\left|u_{m \mathbf{k}, \mathbf{q}}^{\mathcal{E}_{\alpha}}\right\rangle$ and $\left|u_{m \mathbf{k}, \mathbf{q}}^{\lambda}\right\rangle$, 


$$
\begin{aligned}
E_{\mathbf{q}}^{\mathcal{E}_{\alpha}^{*} \lambda}= & s \int_{\mathrm{BZ}}\left[d^{3} k\right] \sum_{m} E_{m \mathbf{k}, \mathbf{q}}^{\mathcal{E}_{\alpha}^{*} \lambda} \\
& +\frac{1}{2} \iint K_{\mathbf{q}}\left(\mathbf{r}, \mathbf{r}^{\prime}\right) n_{\mathbf{q}}^{\mathcal{E}_{\alpha}^{*}}(\mathbf{r}) n_{\mathbf{q}}^{\lambda}\left(\mathbf{r}^{\prime}\right) d^{3} r d^{3} r^{\prime}
\end{aligned}
$$

(we neglect the nonvariational term, as it is generally absent in the case of the $\mathcal{E}$-field response), where

$$
\begin{aligned}
E_{m \mathbf{k}, \mathbf{q}}^{\mathcal{E}_{\alpha}^{*} \lambda}= & \left\langle u_{m \mathbf{k}, \mathbf{q}}^{\mathcal{E}_{\alpha}}\left|\left(\hat{H}_{\mathbf{k}+\mathbf{q}}^{(0)}+a \hat{P}_{\mathbf{k}+\mathbf{q}}-\epsilon_{m \mathbf{k}}\right)\right| u_{m \mathbf{k}, \mathbf{q}}^{\lambda}\right\rangle \\
& +\left\langle u_{m \mathbf{k}, \mathbf{q}}^{\mathcal{E}_{\alpha}}\left|\hat{Q}_{\mathbf{k}+\mathbf{q}} H_{\mathbf{k}, \mathbf{q}}^{\lambda}\right| u_{m \mathbf{k}}^{(0)}\right\rangle+\left\langle i u_{m \mathbf{k}, \mathbf{q}}^{A_{\alpha}} \mid u_{m \mathbf{k}, \mathbf{q}}^{\lambda}\right\rangle .
\end{aligned}
$$

Note that Eq. (36) is not a variational function of the Afield response wave functions $\left|i u_{m \mathbf{k}, \mathbf{q}}^{A_{\alpha}}\right\rangle$. Consequently, when calculating the $\mathbf{q}$ derivatives of $E_{\mathbf{q}}^{\mathcal{E}_{\mathbf{q}}^{*} \lambda}$, one needs to explicitly derive the functions $\left|i u_{m \mathbf{k}, \mathbf{q}}^{A_{\alpha}}\right\rangle$ as one would do for a standard external potential operator (e.g., corresponding to a phonon or a "metric" [26] perturbation, as in the flexoelectric case of Sec. V). Note also that at $\mathbf{q}=0$, the above formulas trivially reduce to the standard treatment of the uniform electric field perturbation [11], of which they constitute the desired generalization to arbitrary $\mathbf{q}$ vectors.

Before closing this subsection, it is interesting to verify where the variational formulas derived here stand compared to the existing treatment of the polarization response [20] via Eq. (28). By imposing the stationary condition Eq. (35) to Eq. (36), we obtain the following nonstationary formula for the polarization response,

$$
\frac{d P_{\alpha}^{\mathbf{q}}}{d \lambda}=-\frac{2}{\Omega} E_{\mathbf{q}}^{\mathcal{E}_{\alpha}^{*} \lambda}=-\frac{2 s}{\Omega} \int_{\mathrm{BZ}}\left[d^{3} k\right] \sum_{m}\left\langle i u_{m \mathbf{k}, \mathbf{q}}^{A_{\alpha}} \mid u_{m \mathbf{k}, \mathbf{q}}^{\lambda}\right\rangle .
$$

It is not difficult to show that Eq. (38) exactly matches Eq. (28). One just needs to recall the relationship between the current-density operator and the vector potential perturbation Eq. (A5) and the sum-over-states expression of the adiabatic wave functions [a consequence of Eq. (34)],

$$
\left|\delta u_{m \mathbf{k}, \mathbf{q}}^{\lambda}\right\rangle=i \sum_{n \in \text { unocc }}\left|u_{n \mathbf{k}+\mathbf{q}}^{(0)}\right\rangle \frac{\left\langle u_{n \mathbf{k}+\mathbf{q}}^{(0)} \mid u_{m \mathbf{k}, \mathbf{q}}^{\lambda}\right\rangle}{\epsilon_{n \mathbf{k}+\mathbf{q}}-\epsilon_{m \mathbf{k}}} .
$$

To go from Eq. (28) to Eq. (38), it suffices then to incorporate Eq. (39) into Eq. (28) and subsequently move the energy denominator and the factor of $i$ from the right $(\lambda$ response) to the left (A response) matrix element. This derivation shows that, apart from irrelevant differences in the notation, Eq. (28) can be regarded as the nonstationary [11] counterpart of the variational functional, Eq. (36).

\section{Scalar potential}

A monochromatic scalar potential perturbation simply involves adding $\varphi e^{i \mathbf{q} \cdot \mathbf{r}}$ to the local electrostatic potential; thus, in the language of this work, the external perturbation is the unity operator at any $\mathbf{q}, \hat{H}_{\mathbf{q}}^{\varphi}=\mathcal{Q}=-1$, where $\mathcal{Q}$ is the electron charge. The mixed derivative functional involving a scalar potential and a second perturbation $\lambda$ then reads as (note, as in the electric field case, the disappearance of the nonvariational term)

$$
\begin{aligned}
E_{\mathbf{q}}^{\varphi^{*} \lambda}= & s \int_{\mathrm{BZ}}\left[d^{3} k\right] \sum_{m} E_{m \mathbf{k}, \mathbf{q}}^{\varphi^{*} \lambda} \\
& +\frac{1}{2} \iint K_{\mathbf{q}}\left(\mathbf{r}, \mathbf{r}^{\prime}\right) n_{\mathbf{q}}^{\varphi *}(\mathbf{r}) n_{\mathbf{q}}^{\lambda}\left(\mathbf{r}^{\prime}\right) d^{3} r d^{3} r^{\prime},
\end{aligned}
$$

where

$$
\begin{aligned}
E_{m \mathbf{k}, \mathbf{q}}^{\varphi^{*} \lambda}= & \left\langle u_{m \mathbf{k}, \mathbf{q}}^{\varphi}\left|\left(\hat{H}_{\mathbf{k}+\mathbf{q}}^{(0)}+a \hat{P}_{\mathbf{k}+\mathbf{q}}-\epsilon_{m \mathbf{k}}\right)\right| u_{m \mathbf{k}, \mathbf{q}}^{\lambda}\right\rangle \\
& -\left\langle u_{m \mathbf{k}}^{(0)}\left|\hat{Q}_{\mathbf{k}+\mathbf{q}}\right| u_{m \mathbf{k}, \mathbf{q}}^{\lambda}\right\rangle+\left\langle u_{m \mathbf{k}, \mathbf{q}}^{\varphi}\left|\hat{Q}_{\mathbf{k}+\mathbf{q}} \hat{H}_{\mathbf{k}, \mathbf{q}}^{\lambda}\right| u_{m \mathbf{k}}^{(0)}\right\rangle .
\end{aligned}
$$

Differentiation with respect to $\left|u_{m \mathbf{k}, \mathbf{q}}^{\lambda}\right\rangle$ yields the Sternheimer equation for the first-order wave functions,

$\left(\hat{H}_{\mathbf{k}+\mathbf{q}}^{(0)}+a \hat{P}_{\mathbf{k}+\mathbf{q}}-\epsilon_{m \mathbf{k}}\right)\left|u_{m \mathbf{k}, \mathbf{q}}^{\varphi}\right\rangle=-\hat{Q}_{\mathbf{k}+\mathbf{q}}\left(-1+\hat{V}_{\mathbf{q}}^{\varphi}\right)\left|u_{m \mathbf{k}}^{(0)}\right\rangle$,

where $\hat{V}_{\mathbf{q}}^{\varphi}$ is, as usual, the SCF contribution to the perturbation.

As we mentioned earlier, the scalar potential response vanishes at $\mathbf{q}=0$, and any mixed derivative involving $\varphi$ identically vanishes at $\mathbf{q}=0$ as well. (Note that the perturbation does not vanish at $\mathbf{q}=0$, as it is a constant equal to -1 at any value of the wave vector.) At first order in $\mathbf{q}$, one recovers the standard treatment of the uniform electric field [22], with the following relationship between the corresponding first-order wave functions,

$$
\left|u_{m \mathbf{k}}^{\mathcal{E}_{\delta}}\right\rangle=\left|i u_{m \mathbf{k}, \delta}^{\varphi}\right\rangle .
$$

Then, by combining Eq. (43) with our higher-order formula Eq. (24), one can obtain useful information about the dispersion of the charge-density response of the system to an arbitrary perturbation.

To see the relationship between the scalar potential and the first-order charge density, one can insert Eq. (42) into Eq. (40) to obtain a nonstationary expression for the mixed derivative,

$$
E_{\mathbf{q}}^{\varphi^{*} \lambda}=-s \int_{\mathrm{BZ}}\left[d^{3} k\right] \sum_{m}\left\langle u_{m \mathbf{k}}^{(0)}\left|\hat{Q}_{\mathbf{k}+\mathbf{q}}\right| u_{m \mathbf{k}, \mathbf{q}}^{\lambda}\right\rangle,
$$

which provides a direct link to the electronic contribution to the charge-density response, 


$$
\bar{\rho}_{\mathrm{el}, \mathbf{q}}^{\lambda}=-\frac{1}{\Omega} \int_{\Omega} d^{3} r n_{\mathbf{q}}^{\lambda}(\mathbf{r})=\frac{2}{\Omega} E_{\mathbf{q}}^{\varphi^{*} \lambda}
$$

Note that $\bar{\rho}_{\text {el,q, }}^{\lambda}$, the cell-averaged electronic charge density induced by the perturbation $\lambda$, differs from the cell average of $n_{\mathbf{q}}^{\lambda}(\mathbf{r})$ by a minus sign, which stems from the negative electron charge.

\section{Relationship to the continuity equation}

The fact that $E_{\mathbf{q}}^{\varphi^{*} \lambda}$ and $-E_{\mathbf{q}}^{\mathcal{E}_{\mathbf{\alpha}}^{*} \lambda}$ correspond, respectively (modulo a factor of $2 / \Omega$ ), to the charge-density and polarization response to the perturbation $\lambda$ implies that they must satisfy the continuity equation $\boldsymbol{\nabla} \cdot \mathbf{P}=-\rho$. In reciprocal space, this means that the following must be true,

$$
i \sum_{\alpha} q_{\alpha} E_{\mathbf{q}}^{\mathcal{E}_{\alpha}^{*} \lambda}=E_{\mathbf{q}}^{\varphi^{*} \lambda} .
$$

The correctness of this result can be, of course, verified at the level of the finite-q functionals, respectively, Eqs. (36) and (40). In the context of the present work, however, it is perhaps more insightful to verify Eq. (46) in the longwave limit and use it as a "sanity check" of the formalism. At the lowest orders in $\mathbf{q}$, Eq. (46) leads to the following relationships,

$$
\begin{gathered}
-E^{\mathcal{E}_{\alpha}^{*} \lambda}=i E_{\alpha}^{\varphi^{*} \lambda}, \\
i E_{\beta}^{\mathcal{E}_{\alpha}^{*} \lambda}+i E_{\alpha}^{\mathcal{E}_{\beta}^{*} \lambda}=-E_{\alpha \beta}^{\varphi^{*} \lambda} .
\end{gathered}
$$

(We choose the prefactors in such a way that all quantities are real numbers and that they match the sign conventions of Ref. [13].) Equation (47a) is trivial to verify by using Eq. (36) and the Hellmann-Feynman theorem applied to the first q gradient of Eq. (40). Equation (47b) can be checked by applying the higher-order formula Eq. (24) to Eq. (40) and by using the relationship existing between the scalar potential and the electric field response functions, Eq. (43). In the special case of $\lambda$ being an atomic displacement, one can recognize the relationships between the multipolar expansion of the charge-density and polarization response as established in Ref. [13].

\section{DYNAMICAL QUADRUPOLE TENSOR}

\section{A. Theory}

Following the notation of Ref. [13], we can define the cell-integrated charge response to a monochromatic atomic displacement as

$$
Q_{\kappa \beta}^{\mathbf{q}}=\Omega \bar{\rho}_{\mathbf{q}}^{\tau_{\kappa \beta}}=-i q_{\beta} Z_{\kappa}+2 E_{\mathbf{q}}^{\varphi^{*} \tau_{\kappa \beta}},
$$

where $Z_{\kappa}$ is the pseudopotential charge, and $2 E_{\mathbf{q}}^{\varphi^{*} \tau_{\kappa \beta}}$ is the mixed derivative of the energy with respect to a scalar potential [see Sec. III C, Eq. (40) in particular] and an atomic displacement pattern of the type

$$
\mathbf{R}_{l \kappa}=\mathbf{R}_{l \kappa}^{0}+\boldsymbol{\tau}_{\kappa} e^{i \mathbf{q} \cdot \mathbf{R}_{l \kappa}^{0}}
$$

( $l$ and $\kappa$ are cell and sublattice indices, respectively. $\mathbf{R}_{l \kappa}^{0}$ indicates the unperturbed atomic position. Note that this perturbation differs from the standard implementations of DFPT [12,22] by a phase factor; see Appendix B 1 for details.)

In the long-wave limit, $Q_{\kappa \beta}^{\mathbf{q}}$ can be written as a multipole expansion of the charge density induced by an atomic displacement,

$$
Q_{\kappa \beta}^{\mathbf{q}}=-i q_{\gamma} Q_{\kappa \beta}^{(1, \gamma)}-\frac{q_{\gamma} q_{\delta}}{2} Q_{\kappa \beta}^{(2, \gamma \delta)}+\cdots,
$$

where the dots stand for higher-order terms that we do not discuss in this work. The first-order term corresponds to the Born effective charge tensor,

$$
Z_{\kappa, \beta \gamma}^{*}=Q_{\kappa \beta}^{(1, \gamma)}=\delta_{\beta \gamma} Z_{\kappa}+\Delta Z_{\kappa, \beta \gamma},
$$

where the electronic contribution reads as

$$
\begin{aligned}
\Delta Z_{\kappa, \beta \gamma}= & =2 i s \int_{\mathrm{BZ}}\left[d^{3} k\right] \sum_{m}\left\langle u_{m \mathbf{k}}^{(0)}\left|\partial_{\gamma} \hat{P}_{\mathbf{k}}\right| u_{m \mathbf{k}}^{\tau_{\kappa \beta}}\right\rangle \\
& =2 i s \int_{\mathrm{BZ}}\left[d^{3} k\right] \sum_{m}\left\langle\tilde{\partial}_{\gamma} u_{m \mathbf{k}}^{(0)} \mid u_{m \mathbf{k}}^{\tau_{\kappa \beta}}\right\rangle \\
& =-2 s \int_{\mathrm{BZ}}\left[d^{3} k\right] \sum_{m}\left\langle i \tilde{\partial}_{\gamma} u_{m \mathbf{k}}^{(0)} \mid u_{m \mathbf{k}}^{\tau_{\kappa \beta}}\right\rangle,
\end{aligned}
$$

thus, recovering the already established result $[11,12]$. [We apply the Hellmann-Feynman theorem to the $\mathbf{q}$ derivative of the functional of Eq. (40), combined with the fact that the $\varphi$-response wave functions vanish at $\mathbf{q}=0$.]

The quadrupole tensor elements can be written as the second $\mathbf{q}$ gradients of $Q_{\kappa \beta}^{\mathbf{q}}$,

$$
Q_{\kappa \beta}^{(2, \gamma \delta)}=-2 E_{\gamma \delta}^{\varphi^{*} \tau_{\kappa \beta}} .
$$

By using Eq. (47b), we arrive at the following expression,

$$
E_{\gamma \delta}^{\varphi^{*} \tau_{\kappa \beta}}=-i E_{\gamma}^{\mathcal{E}_{\delta}^{*} \tau_{\kappa \beta}}-i E_{\delta}^{\mathcal{E}_{\gamma}^{*} \tau_{\kappa \beta}} .
$$

The first $\mathbf{q}$ gradient of the mixed response to an electric field and to an atomic displacement can then be calculated by applying Eqs. (16) and (17) to Eqs. (36) and (37), respectively,

$$
\begin{aligned}
E_{\gamma}^{\mathcal{E}_{\delta}^{*} \tau_{\kappa \beta}}= & s \int_{\mathrm{BZ}}\left[d^{3} k\right] \sum_{m} E_{m \mathbf{k}, \gamma}^{\mathcal{E}_{\delta}^{*} \tau_{\kappa \beta}} \\
& +\frac{1}{2} \int_{\Omega} \int K_{\gamma}\left(\mathbf{r}, \mathbf{r}^{\prime}\right) n^{\mathcal{E}_{\delta}}(\mathbf{r}) n^{\tau_{\kappa \beta}}\left(\mathbf{r}^{\prime}\right) d^{3} r d^{3} r^{\prime}
\end{aligned}
$$

with 


$$
\begin{aligned}
E_{m \mathbf{k}, \gamma}^{\mathcal{E}_{\delta}^{*} \tau_{\kappa \beta}}= & \left\langle u_{m \mathbf{k}}^{\mathcal{E}_{\delta}}\left|\partial_{\gamma} \hat{H}_{\mathbf{k}}^{(0)}\right| u_{m \mathbf{k}}^{\tau_{\kappa \beta}}\right\rangle+\left\langle u_{m \mathbf{k}}^{\mathcal{E}_{\delta}}\left|\partial_{\gamma} \hat{Q}_{\mathbf{k}} \hat{\mathcal{H}}_{\mathbf{k}}^{\tau_{\kappa \beta}}\right| u_{m \mathbf{k}}^{(0)}\right\rangle \\
& +\left\langle u_{m \mathbf{k}}^{(0)}\left|V^{\mathcal{E}_{\delta}} \partial_{\gamma} \hat{Q}_{\mathbf{k}}\right| u_{m \mathbf{k}}^{\tau_{k \beta}}\right\rangle+\left\langle u_{m \mathbf{k}}^{\mathcal{E}_{\delta}}\left|\hat{H}_{\mathbf{k}, \gamma}^{\tau_{\kappa \beta}}\right| u_{m \mathbf{k}}^{(0)}\right\rangle \\
& +\left\langle i u_{m \mathbf{k}, \gamma}^{A_{\delta}} \mid u_{m \mathbf{k}}^{\tau_{\kappa \beta}}\right\rangle .
\end{aligned}
$$

Since the response functions need to be symmetrized according to Eq. (54), we can simplify the expression of $\left|u_{m \mathbf{k}, \gamma}^{A_{\delta}}\right\rangle$, Eq. (32), and set

$$
\left\langle i u_{m \mathbf{k}, \gamma}^{A_{\delta}} \mid u_{m \mathbf{k}}^{\tau_{\kappa \beta}}\right\rangle \rightarrow-\frac{i}{2}\left\langle u_{m \mathbf{k}}^{(0)}\left|\partial_{\gamma \delta} \hat{P}_{\mathbf{k}}\right| u_{m \mathbf{k}}^{\tau_{\kappa \beta}}\right\rangle,
$$

where $\partial_{\gamma \delta} \hat{P}_{\mathbf{k}}$ is the second $\mathbf{k}$ gradient of the valence-band projector that we describe in Appendix A. [Other terms in Eq. (32) vanish as they are antisymmetric in the two indices.] The explicit formula for the $\mathbf{q}$ derivative of the atomic displacement perturbation $\hat{H}_{\mathbf{k}, \gamma}^{\tau_{\kappa \beta}}$ is reported in Appendix B 1.

Note that we can obtain the same result by directly applying the higher-order formula Eq. (24) to Eq. (40), instead of using Eq. (54). The above procedure has the advantage that the intermediate quantity $E_{m \mathbf{k}, \gamma}^{\mathcal{E}_{\tilde{\delta}}^{*} \tau_{\kappa \beta}}$ has also a well-defined physical meaning, as it relates to the $\mathbf{P}^{(1)}$ tensors discussed in Refs. [6,13],

$$
P_{\delta, \kappa \beta}^{(1, \gamma)}=\frac{2 i}{\Omega} E_{\gamma}^{\mathcal{E}_{\delta}^{*} \tau_{\kappa \beta}} .
$$

These quantities can be interpreted as the first spatial moment of the polarization field induced by an atomic displacement and are required for the calculation of the socalled "mixed" contribution to the flexoelectric tensor. Their in-depth discussion brings us out of our main topic, and we defer it to a forthcoming publication.

\section{B. Computational parameters}

The computation of the quadrupole tensor is implemented in the ABINIT [29] package as a postprocessing of the DFPT response function calculations. All the numerical results are obtained employing Troullier-Martins normconserving pseudopotentials and the Perdew-Wang [30] parametrization of the LDA. For our calculations on bulk $\mathrm{Si}$, we use the calculated cell parameter of $a_{0}=10.102$ bohr and two different crystal cells: (i) the primitive twoatom cell sampled with a Monkhorst-Pack (MP) mesh of $12 \times 12 \times 12 \mathbf{k}$ points, and (ii) a nonprimitive six-atom hexagonal cell with the translation vectors oriented along the $[01 \overline{1}],[10 \overline{1}$,$] and [111]$ directions, sampled with a $\Gamma$ centered $22 \times 22 \times 22 \mathbf{k}$ mesh. We use a plane-wave cutoff of $20 \mathrm{Ha}$ in both cell types. We also perform a convergence study by repeating our calculations at several different values of the cutoff and $\mathbf{k}$-point mesh resolution. Regarding our calculations of ferroelectric $\mathrm{PbTiO}_{3}$, we use a tetragonal five-atom unit cell, with a plane-wave cutoff of $70 \mathrm{Ha}$ and an $8 \times 8 \times 8$ MP mesh of $\mathbf{k}$ points. We relax the unit cell until the forces are smaller than $1 \times 10^{-6} \mathrm{Ha} / \mathrm{bohr}$, obtaining an aspect ratio of $c / a=1.046(a=7.275 \mathrm{bohr})$ and a spontaneous polarization $P_{S}=0.78 \mathrm{C} / \mathrm{m}^{2}$. These structural data are in excellent agreement with earlier calculations of the same system [27].

\section{Numerical results}

We first study bulk $\mathrm{Si}$ as a test case. The quadrupolar tensor is defined by a single material constant $Q$ via the following expression,

$$
Q_{\kappa \beta}^{(2, \gamma \delta)}=(-1)^{\kappa+1} Q\left|\varepsilon_{\beta \gamma \delta}\right|,
$$

where $\varepsilon_{\beta \gamma \delta}$ is the Levi-Civita tensor. In order to benchmark the formalism, we first perform a calculation of $Q$ via an independent real-space method, which does not rely on Eq. (53). To this end, we calculate the charge-density response to an atomic displacement along the [111] direction by using a Brillouin-zone unfolding procedure [31] applied to the six-atom hexagonal cell. In particular, we consider a stripe of 22 equidistant $\mathbf{q}$ points $(\mathbf{q}=0$ included), spanning the entire Brillouin zone along the crystallographic [111] direction and calculate the first-order densities associated with a phonon perturbation at each $\mathbf{q}$. (In practice, the number of independent $\mathbf{q}$ points reduces to 12 due to time-reversal symmetry.) After unfolding, we readily obtain the induced charge density that corresponds to a displacement of an isolated atom. (In practice, this approach corresponds to studying the displacement of a plane of atoms in a supercell where the hexagonal unit is repeated 22 times along the [111] direction.) We report the plane averages of the first-order density in Fig. 1, where we also show its decomposition into the antisymmetric and symmetric contributions. A fast decay of the induced density is clearly observed, which allows us to calculate the desired real-space moments with high numerical accuracy. The dipole moment correctly reproduces the pseudopotential charge, as expected. The second real-space moment of the first-order charge is then related to $Q$ via

$$
Q^{\mathrm{RS}}=\frac{\sqrt{3}}{2} \epsilon_{\infty} \bar{Q}_{[111]}^{(2)},
$$

where the superscript RS stands for "real space," and $\epsilon_{\infty}$ is the calculated electronic dielectric constant.

Our calculated values are $\bar{Q}_{[111]}^{(2)}=1.178$ and $\epsilon_{\infty}=13.103$, which via Eq. (60) yield a value of $Q^{\mathrm{RS}}=13.367 e$ bohr. With an equivalent choice of the computational parameters, by using our new method, Eq. (53) and the primitive twoatom cell, we obtain $Q=13.368 e$ bohr. The matching between the two approaches is essentially perfect, which demonstrates the soundness of our implementation. 


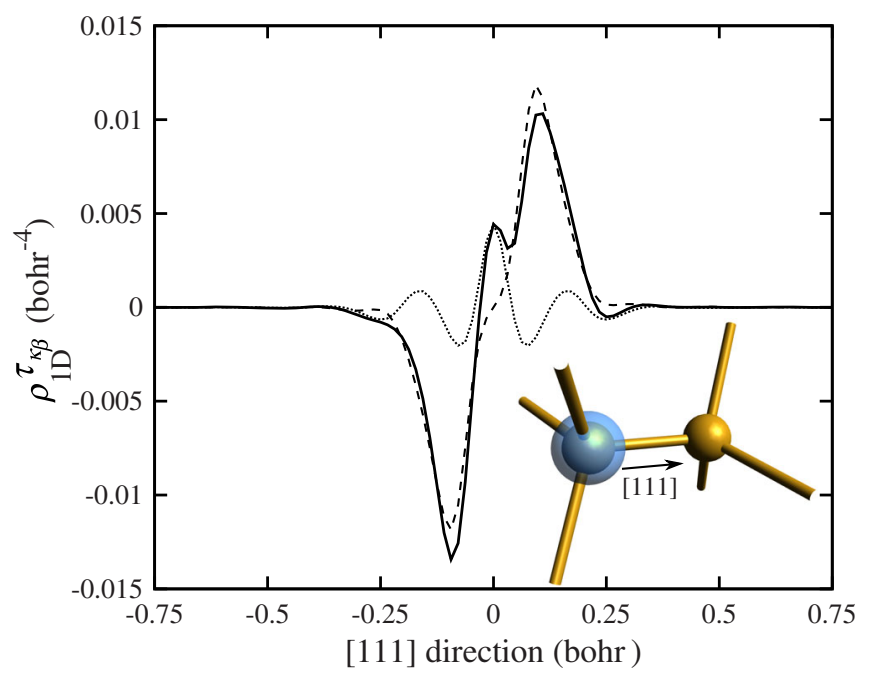

FIG. 1. Electron-density response to an atomic displacement along the [111] direction in bulk Si (solid line). Its symmetric (dotted line) and antisymmetric (dashed line) parts are also plotted. The first-order density is averaged in plane. The origin of the abscissas coincides with the position of the atomic sublattice highlighted in the inset.

To better illustrate the plane-wave and k-point mesh requirements of our new method, we also perform a convergence study, where we compare the behavior of the quadrupole tensor components (alongside with the flexoelectric response, which we comment on in Sec. V) to that of a standard linear-response quantity, the electronic dielectric constant. The numerical results are plotted in Figs. 2(a) and 2(b) as a function of the plane-wave energy cutoffs and the number of $\mathbf{k}$ points employed to sample the Brillouin zone. One can clearly appreciate from the figure that the quadrupoles [Fig. 2(a)] and the dielectric constant
[Fig. 2(b)] converge equally fast with respect to both computational parameters. Moreover, the agreement between $Q$ and $Q^{\mathrm{RS}}$ becomes better and better as the energy cutoff increases. Both observations concur to put our new method based on Eq. (53) on very firm ground. Note that the calculation via Eq. (53) is at least an order of magnitude more efficient than the alternative real-space method, as the latter requires us to calculate the phonon response at many $\mathbf{q}$ points, while the present approach requires only $\Gamma$-point response functions as prerequisites.

Next, as a more ambitious test of our method, we carry out a numerical verification of Martin's formula [14]

$$
e_{\alpha \beta \gamma}=-\frac{1}{2 \Omega} \sum_{\kappa}\left(Q_{\kappa \beta}^{(2, \alpha \gamma)}-Q_{\kappa \alpha}^{(2, \gamma \beta)}+Q_{\kappa \gamma}^{(2, \beta \alpha)}\right),
$$

relating the proper [32] clamped-ion piezoelectric tensor $e_{\alpha \beta \gamma}$ to the sublattice sum of the dynamical quadrupoles. [Here, the first subscript $(\alpha)$ of the piezoelectric tensor on the left-hand side indicates the polarization direction, whereas the other two indices $(\beta \gamma)$ refer to the strain tensor components.] In particular, we benchmark the value of $e_{\alpha \beta \gamma}$ computed from Eq. (61) via the quadrupoles against its value obtained as the mixed derivative of the energy with respect to the components of the strain and the electric field. The latter response function is a standard DFPT quantity that we obtain by means of the metric tensor formulation by Hamann et al. [33] as implemented in the ABINIT package.

We focus on a well-known piezoelectric system, the tetragonal phase of $\mathrm{PbTiO}_{3}$. The quadrupole moments of each atom in the unit cell are shown in Table I. As for the three independent $\mathrm{PbTiO}_{3}$ piezoelectric tensor elements, they are reported in Table II. The comparison between the coefficients from the two methods demonstrates

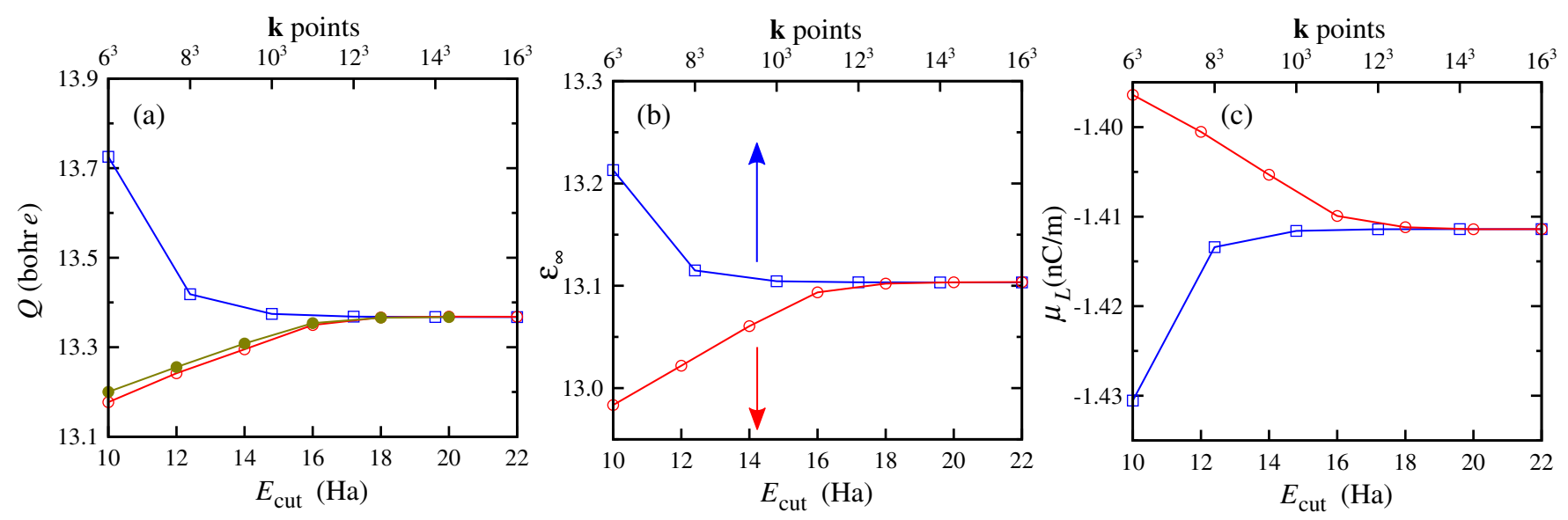

FIG. 2. Convergence of selected linear-response quantities with respect to the plane-wave cutoff and the density of the $\mathbf{k}$-point mesh. Calculated dynamic quadrupole moment (a), dielectric constant (b) and longitudinal flexoelectric coefficient (c) are shown. Red empty circles (lines are a guide to the eye) are obtained by varying the plane-waves energy cutoff while keeping the $12 \times 12 \times 12 \mathbf{k}$-points grid fixed. Empty blue squares correspond to varying the k-points mesh resolution with a fixed energy cut-off of $20 \mathrm{Ha}$. Panel (a) includes the energy cut-off dependence of the quadrupole constant as calculated from the second moment of the induced charge density response (green circles). 
TABLE I. Quadrupole moments (in $e$ bohr) of $\mathrm{PbTiO}_{3}$ calculated via Eq. (53). Note that $Q_{\kappa \alpha}^{(2, \beta \gamma)}=Q_{\kappa \alpha}^{(2, \gamma \beta)}$.

\begin{tabular}{lrrrrr}
\hline \hline & $\kappa=\mathrm{Pb}$ & $\kappa=\mathrm{Ti}$ & $\kappa=\mathrm{O}_{1}$ & $\kappa=\mathrm{O}_{2}$ & $\kappa=\mathrm{O}_{3}$ \\
\hline$Q_{\kappa 3}^{(2,11)}$ & 2.264 & -3.545 & 2.884 & -4.186 & 0.406 \\
$Q_{\kappa 3}^{(2,22)}$ & 2.264 & -3.545 & -4.186 & 2.884 & 0.406 \\
$Q_{\kappa 1}^{(2,31)}$ & -0.062 & -3.799 & 3.123 & -1.115 & -1.784 \\
$Q_{\kappa 2}^{(2,32)}$ & -0.062 & -3.799 & -1.115 & 3.123 & -1.784 \\
$Q_{\kappa 3}^{(2,33)}$ & 1.240 & -0.195 & 2.027 & 2.027 & 6.653 \\
\hline \hline
\end{tabular}

TABLE II. Clamped-ion piezoelectric coefficients (in $\mathrm{C} / \mathrm{m}^{2}$ ) of $\mathrm{PbTiO}_{3}$ calculated via two different methods. "Strain": Standard DFPT approach relying on the strain [33] and electric field response. "Quadrupoles": From the quadrupole moments via Eq. (61). Literature values from Ref. [34] are shown for comparison.

\begin{tabular}{lccc}
\hline \hline & $e_{113}=e_{223}$ & $e_{311}=e_{322}$ & $e_{333}$ \\
\hline Strain & 0.1547 & 0.3617 & -0.8345 \\
Quadrupoles & 0.1548 & 0.3614 & -0.8347 \\
Ref. [34] & 0.20 & 0.35 & -0.88 \\
\hline \hline
\end{tabular}

exceptionally good agreement, which improves up to the fifth decimal digit by increasing the density of $\mathbf{k}$ points to $14 \times 14 \times 14$. Our results also qualitatively agree with those of Ref. [34], wherein a different set of lattice parameters, pseudopotentials, and exchange-correlation functionals were employed.

\section{FLEXOELECTRICITY}

\section{A. Theory}

The flexoelectric tensor describes the macroscopic polarization response to a strain gradient. It can be written in two different ways $[6,13]$ depending on how the straingradient tensor is defined. The type-I form is

$$
\mu_{\alpha \beta, \gamma \delta}^{\mathrm{I}}=\frac{d P_{\alpha}}{d \eta_{\beta, \gamma \delta}},
$$

where $\eta_{\beta, \gamma \delta}$ is the second spatial gradient of the displacement field. Alternatively, one can use the type-II form,

$$
\mu_{\alpha \gamma, \beta \delta}^{\mathrm{II}}=\frac{d P_{\alpha}}{d \varepsilon_{\beta \delta, \gamma}},
$$

where $\varepsilon_{\beta \delta, \gamma}$ is the first gradient of the symmetric strain tensor. The two representations contain the same information and are symmetric under permutation of the last two indices. One can convert one into the other and vice versa via the following relationships

$$
\begin{gathered}
\mu_{\alpha \gamma, \beta \delta}^{\mathrm{II}}=\mu_{\alpha \beta, \gamma \delta}^{\mathrm{I}}+\mu_{\alpha \delta, \beta \gamma}^{\mathrm{I}}-\mu_{\alpha \gamma, \delta \beta}^{\mathrm{I}}, \\
\mu_{\alpha \beta, \gamma \delta}^{\mathrm{I}}=\frac{1}{2}\left(\mu_{\alpha \gamma, \beta \delta}^{\mathrm{II}}+\mu_{\alpha \delta, \beta \gamma}^{\mathrm{II}}\right) .
\end{gathered}
$$

From the point of view of atomistic calculations, flexoelectricity can be decomposed into three distinct contributions [13]: lattice mediated, mixed, and electronic. In principle, all three can be written by using the formalism developed in this work in terms of a few basic ingredients. These ingredients are the mixed response to an electric field, atomic displacement, or metric wave perturbation taken at first or second order in $\mathbf{q}$. We defer the detailed implementation and test of the full flexoelectric tensor to a forthcoming publication and focus here on the purely electronic response only.

The electronic flexoelectric tensor can be written as the second derivative with respect to $\mathbf{q}$ of the current density that is adiabatically induced by a "clamped-ion" acoustic phonon perturbation [20], i.e., to a displacement pattern of the type

$$
\mathbf{R}_{l \kappa}=\mathbf{R}_{l \kappa}^{0}+\mathbf{u} e^{i \mathbf{q} \cdot \mathbf{R}_{l \kappa}^{0}} .
$$

Note the absence of the basis index on the perturbation parameter; this implies that all atoms in the primitive cell should be displaced simultaneously with equal amplitude u. Thus, a calculation of the flexoelectric tensor can be, in principle, carried out by regarding Eq. (65) as the sublattice sum of Eq. (49), which leads to the following practical scheme. First, one writes the polarization response to the displacement of an individual sublattice at finite $\mathbf{q}$; then, a second-order expansion in the wave vector $\mathbf{q}$ is performed; finally, the clamped-ion flexoelectric tensor is written as a sublattice sum of the result [13]. This was indeed the strategy adopted in Ref. [20].

In the context of this work, however, such an approach is impractical - the phonon perturbation of Eq. (49) does not vanish in the $\mathbf{q}=0$ limit. Therefore, Eq. (24) cannot be directly applied to calculate expansion to second order in q of the corresponding polarization response. To work around this obstacle, we follow Refs. [26,35] and recast the acoustic phonon as a "metric wave" perturbation by operating a coordinate transformation to the curvilinear comoving frame. We then write the polarization response to the acoustic phonon at finite $\mathbf{q}$ as (following the notation of Ref. [26])

$$
\bar{P}_{\alpha, \beta}^{\prime \mathbf{q}}=-\frac{2}{\Omega} E_{\mathbf{q}}^{\mathcal{E}_{\alpha}^{*}(\beta)}
$$

where $E_{\mathbf{q}}^{\mathcal{E}_{\alpha}^{*}(\beta)}$ refers to the mixed derivative of Eq. (36) specialized to the case $\lambda=(\beta)$, and $(\beta)$ indicates a metric wave with the displacement field oriented along the Cartesian direction $\beta$. (The overline implies cell averaging, 
and the prime indicates that we implicitly discard the magneticlike contribution from rotation gradients, following the arguments of Refs. [20,26,35].)

It is useful at this stage to recall [26] two crucial properties of the metric wave: (i) both the perturbation and the response vanish at $\mathbf{q}=\mathbf{0}$,

$$
\hat{H}_{\mathbf{k}, \mathbf{q}=\mathbf{0}}^{(\beta)}=0, \quad\left|u_{m \mathbf{k}, \mathbf{q}=\mathbf{0}}^{(\beta)}\right\rangle=0 ;
$$

(ii) at first order in $\mathbf{q}$, the metric wave reduces to the uniform strain perturbation $\eta_{\beta \gamma}$ by Hamann et al. [33],

$$
\hat{H}_{\mathbf{k}, \delta}^{(\beta)}=i \hat{H}_{\mathbf{k}}^{\eta_{\beta \delta}}, \quad\left|u_{m \mathbf{k}, \delta}^{(\beta)}\right\rangle=i\left|u_{m \mathbf{k}}^{\eta_{\beta \delta}}\right\rangle .
$$

As we see shortly, properties (i) and (ii) allow us to write down a closed expression for the clamped-ion flexoelectric tensor by using the second-order formula, Eq. (24). Before doing that, it is useful to perform a consistency check of Eq. (66) by showing that it correctly recovers the piezoelectric tensor at first order in $\mathbf{q}$. The clamped-ion piezoelectric tensor can be defined as

$$
e_{\alpha \beta \gamma}=-\left.i \frac{d}{d q_{\gamma}}\left(\frac{d P_{\alpha}^{\mathbf{q}}}{d u_{\beta}}\right)\right|_{\mathbf{q}=\mathbf{0}}=i \frac{2}{\Omega} E_{\gamma}^{\mathcal{E}_{\alpha}^{*}(\beta)}
$$

By applying the Hellmann-Feynman theorem to Eq. (36) and by using Eqs. (67) and (68), we readily obtain

$$
\begin{aligned}
e_{\alpha \beta \gamma} & =i \frac{2 s}{\Omega} \int_{\mathrm{BZ}}\left[d^{3} k\right] \sum_{m}\left\langle u_{m \mathbf{k}}^{\mathcal{E}_{\alpha}}\left|\hat{H}_{\mathbf{k}, \gamma}^{(\beta)}\right| u_{m \mathbf{k}}^{(0)}\right\rangle \\
& =-\frac{2 s}{\Omega} \int_{\mathrm{BZ}}\left[d^{3} k\right] \sum_{m}\left\langle u_{m \mathbf{k}}^{\mathcal{E}_{\alpha}}\left|\hat{H}_{\mathbf{k}}^{\eta_{\beta \gamma}}\right| u_{m \mathbf{k}}^{(0)}\right\rangle,
\end{aligned}
$$

which matches the established result [11,33].

The type-I clamped-ion flexoelectric tensor can now be written in terms of the following formula,

$$
\mu_{\alpha \beta, \gamma \delta}^{\mathrm{I}}=\frac{1}{\Omega} E_{\gamma \delta}^{\mathcal{E}_{\alpha}^{*}(\beta)},
$$

where the mixed derivative is, as above, taken with respect to an electric field and the metric wave perturbation. By taking again into account the relationships existing between the metric $(\beta)$ and the strain perturbations $\eta_{\beta \delta}$, the formulas for the second gradient, Eqs. (24) and (25), are as follows:

$$
\begin{aligned}
\tilde{E}_{\gamma \delta}^{\mathcal{E}}(\beta)= & s \int_{\mathrm{BZ}}\left[d^{3} k\right] \sum_{m} \tilde{E}_{m \mathbf{k}, \gamma \delta}^{\mathcal{E}_{\alpha}^{*}(\beta)} \\
& +\underbrace{\frac{i}{2} \int_{\Omega} \int K_{\gamma}\left(\mathbf{r}, \mathbf{r}^{\prime}\right) n^{\mathcal{E}_{\alpha}}(\mathbf{r}) n^{\eta_{\beta \delta}}\left(\mathbf{r}^{\prime}\right) d^{3} r d^{3} r^{\prime}}_{T_{\text {elst }}},
\end{aligned}
$$

$$
\begin{aligned}
\tilde{E}_{m \mathbf{k}, \gamma \delta}^{\mathcal{E}_{\alpha}^{*}(\beta)}= & \underbrace{i\left\langle u_{m \mathbf{k}}^{\mathcal{E}_{\alpha}}\left|\partial_{\gamma} \hat{H}_{\mathbf{k}}^{(0)}\right| u_{m \mathbf{k}}^{\eta_{\beta \delta}}\right\rangle}_{T_{1}} \\
& +\underbrace{i\left\langle u_{m \mathbf{k}}^{\mathcal{E}_{\alpha}}\left|\partial_{\gamma} \hat{Q}_{\mathbf{k}} \hat{\mathcal{H}}_{\mathbf{k}}^{\eta_{\beta \delta}}\right| u_{m \mathbf{k}}^{(0)}\right\rangle}_{T_{2}}+\underbrace{i\left\langle u_{m \mathbf{k}}^{(0)}\left|\hat{V}^{\mathcal{E}_{\alpha}} \partial_{\gamma} \hat{Q}_{\mathbf{k}}\right| u_{m \mathbf{k}}^{\eta_{\beta \delta}}\right\rangle}_{T_{3}} \\
& +\underbrace{\frac{1}{2\left\langle u_{m \mathbf{k}}^{\mathcal{E}_{\alpha}}\left|\hat{H}_{\mathbf{k}, \gamma \delta}^{(\beta)}\right| u_{m \mathbf{k}}^{(0)}\right\rangle}}_{T_{4}}+\underbrace{i\left\langle i u_{m \mathbf{k}, \gamma}^{A_{\alpha}} \mid u_{m \mathbf{k}}^{\eta_{\beta \delta}}\right\rangle}_{T_{5}}
\end{aligned}
$$

Here we label, for later reference, the five different terms of the band- and $\mathbf{k}$-resolved contribution as $T_{1-5}$, and the term deriving from the self-consistent energy via the gradient of the Coulomb kernel as $T_{\text {elst }}$. Most of the symbols are selfexplanatory, as we already encounter them in the formula for the quadrupolar response. Similar to the quadrupole case, we use Eq. (57) to simplify $T_{5}$; this is justified here because all our tests are performed on cubic materials, where $T_{5}$ must be symmetric with respect to $\alpha \gamma$. The formulas for the $\mathbf{q}$ derivatives of metric perturbation $\hat{H}_{\mathbf{k}, \gamma \delta}^{(\beta)}$ are elaborated in Appendix B 2.

In the following subsection, we present our numerical results in type-II form by using Eq. (64a) whenever appropriate. In practice, this transformation needs to be performed explicitly only on $T_{4}$, since all the other terms are most naturally written in type-II form. (The explicit formula is reported in Appendix B 2.) As we are dealing with cubic crystals only, we adopt the shorthand notation $\mu_{L}=\mu_{11,11}^{\mathrm{II}}, \mu_{T}=\mu_{11,22}^{\mathrm{II}}$, and $\mu_{S}=\mu_{12,12}^{\mathrm{II}}$ for the three independent components, respectively: longitudinal $(L)$, transverse $(T)$, and shear $(S)$. We drop the "II" superscript and assume that the flexoelectric tensor is in type-II form henceforth.

\section{B. Computational parameters}

The computation of the clamped-ion flexoelectric tensor is also implemented in the ABINIT [29] package. The numerical results are obtained with the same type of pseudopotentials and XC functional as in Sec. IV B. For our calculations on noble-gas atoms $\mathrm{He}, \mathrm{Ar}$, and $\mathrm{Kr}$, we use a large cell of $14 \times 14 \times 14$ a.u., with a plane-wave cutoff of $90 \mathrm{Ha}$ and a $2 \times 2 \times 2(4 \times 4 \times 4)$ mesh of $\mathbf{k}$ points to sample the Brillouin zone of $\mathrm{He}$ and $\mathrm{Ar}$ (of $\mathrm{Kr}$ ). For our calculations on $\mathrm{SrTiO}_{3}$, we use a cubic five-atom unit cell with an optimized cell parameter of $a_{0}=7.267 \mathrm{bohr}$, with a plane-wave cutoff of $70 \mathrm{Ha}$ and an $8 \times 8 \times 8$ mesh of $\mathbf{k}$ points. Regarding $\mathrm{Si}$, we use the two-atom primitive cell with the same computational parameters as we describe in Sec. IV B. We also perform a convergence study of the calculated Si flexoelectric tensor by varying the cutoff and $\mathbf{k}$-point mesh resolution. 
TABLE III. Flexoelectric coefficients (in $\mathrm{pC} / \mathrm{m}$ ) of noble-gas atom systems.

\begin{tabular}{lccl}
\hline \hline & $\mu_{L}$ & $\mu_{T}$ & \multicolumn{1}{c}{$\mu_{S}\left(\times 10^{-4}\right)$} \\
\hline $\mathrm{He}$ & $-0.479\left(-0.479^{\mathrm{a}}\right)$ & $-0.479\left(-0.479^{\mathrm{a}}\right)$ & $-0.08\left(-0.08^{\mathrm{a}}\right)$ \\
$\mathrm{Ar}$ & $-4.821\left(-4.813^{\mathrm{a}}\right)$ & $-4.823\left(-4.820^{\mathrm{a}}\right)$ & $-1\left(-10^{\mathrm{a}}\right)$ \\
$\mathrm{Kr}$ & $-6.471\left(-6.474^{\mathrm{a}}\right)$ & $-6.477\left(-6.476^{\mathrm{a}}\right)$ & $-4\left(-20^{\mathrm{a}}\right)$ \\
\hline \hline
\end{tabular}

${ }^{\mathrm{a}}$ Reference [26].

\section{Numerical results}

In order to test our method, we first study a simple cubic crystal lattice consisting of isolated noble-gas atoms, as already investigated in Refs. [6,18,20,26,35]. This toy model presents the advantage that its flexoelectric coefficients can be determined analytically, based on the macroscopic dielectric tensor and the second real-space moment of the unperturbed atomic charge. In particular, the three independent flexoelectric coefficients as calculated from a metric wave perturbation must fulfill the conditions $[26,35] \mu_{L}=\mu_{T}$ and $\mu_{S}=0$.

Table III shows the flexoelectric coefficients calculated for $\mathrm{He}, \mathrm{Ar}$, and $\mathrm{Kr}$. It is clear from the reported data that the expected relationships are satisfied to a high degree of accuracy, and our coefficients are in excellent agreement with those obtained in previous works [26]. The largest deviation is shown by Kr: Being it a larger atom, the overlap between neighboring images is likely to be more pronounced than in the other cases, which justifies the discrepancies we observe with respect to the expectations of the isolated atom model.

At this stage, it is worth emphasizing that such a test is by no means trivial; on the contrary, it represents a very stringent benchmark for our formalism. To demonstrate this point, in Table IV we show a breakdown of the three independent coefficients of the Ar-based crystal into the contributions of the individual terms appearing in Eqs. (72) and (73). The data in the table show a much more complex behavior than the final results of Table III suggest. In particular, the conditions $\mu_{L}=\mu_{T}$ and $\mu_{S}=0$ are not fulfilled by any of the individual terms (an exception is $T_{2}$, but it contributes only a tiny fraction of the final value); instead, the cancellation of the shear component and the equality between the transverse and longitudinal ones both result from a subtle balance between all the terms. Since each term involves a different combination of the input response functions and of the perturbations, such an

TABLE IV. Contribution to the Ar flexoelectric coefficients (in $\mathrm{pC} / \mathrm{m}$ ) from the different terms of Eqs. (72) and (73).

\begin{tabular}{rrrrrrr}
\hline \hline & $T_{\text {elst }}$ & $T_{1}$ & $T_{2}$ & $T_{3}$ & $T_{4}$ & $T_{5}$ \\
\hline$\mu_{L}$ & -6.472 & 3.512 & -0.257 & 1.909 & -9.367 & 5.854 \\
$\mu_{T}$ & -1.885 & -2.286 & -0.257 & 0.000 & -0.395 & 0.000 \\
$\mu_{S}$ & -1.885 & 2.907 & 0.000 & 0.954 & -4.903 & 2.926 \\
\hline \hline
\end{tabular}

TABLE V. Flexoelectric coefficients (in $\mathrm{nC} / \mathrm{m}$ ) of $\mathrm{Si}$ and $\mathrm{SrTiO}_{3}$ along with previous values found in the literature.

\begin{tabular}{llrr}
\hline \hline & $\mu_{L}$ & $\mu_{T}$ & $\mu_{S}$ \\
\hline Si (this work) & -1.4114 & -1.0491 & -0.1895 \\
Ref. [26] & -1.4110 & -1.0493 & -0.1894 \\
$\mathrm{SrTiO}_{3}$ (this work) & -0.8848 & -0.8262 & -0.0823 \\
Ref. [26] $_{\text {Ref. [19] }}$ & -0.8851 & -0.8260 & -0.0823 \\
\hline \hline
\end{tabular}

accurate compensation clearly demonstrates the robustness of the numerical implementation.

We also calculate the electronic contribution to the flexoelectric tensor of two real materials $\mathrm{Si}$ and $\mathrm{SrTiO}_{3}$. The converged values of the flexoelectric coefficients are shown in Table $\mathrm{V}$, where we also compare them to the relevant literature data $[19,20,26]$. Again, the excellent agreement with the published values is clear. Nevertheless, we stress that our results are obtained with a small fraction of the computational effort that was formerly needed. Indeed, the most efficient method to calculate the flexoelectric tensor prior to this work [26] was based on a finitedifference implementation of the long-wave expansion. This required several metric wave response calculations to be performed on a mesh of $\mathbf{q}$ points close to $\Gamma$. As a consequence, it implied a numerical workload that was about 1 order of magnitude larger than the present method, which requires only $\Gamma$-point response functions as an input. (The response at finite $\mathbf{q}$ is much more costly than at $\Gamma$ and needs to be repeated for several q's to perform the numerical differentiations described in Ref. [26].) Also note that, in Ref. [26], the q-mesh spacing is a further numerical parameter that needs to be monitored and controlled, resulting in additional overhead that is associated with the corresponding convergence studies. Nevertheless, the method of Ref. [26] is still orders of magnitude more efficient than the earlier calculations of Ref. [19], which used a supercell geometry to access the transverse components of the bulk tensor.

As a final benchmark, we study the convergence of the flexoelectric coefficients of $\mathrm{Si}$ as a function of the $\mathbf{k}$-point mesh resolution and of the plane-wave cutoff. The results for $\mu_{L}$ are shown in Fig. 2(c). (The convergence of the two other independent components is qualitatively similar to the longitudinal one.) Analogous to the case of the quadrupoles, the flexoelectric coefficients converge at the same rate as the dielectric tensor. This means that all the spatial dispersion properties that we calculate in this work require a computational effort that is comparable to the study of other standard linear-response quantities, such as the electronic dielectric tensor.

\section{CONCLUSIONS AND OUTLOOK}

We establish a general method to perform a systematic study of spatial dispersion effects in the framework of 
density-functional perturbation theory. As a practical demonstration, we implement the dynamical quadrupole tensor and the clamped-ion flexoelectric tensor in the ABINIT package and perform extensive numerical tests. This work opens a number of exciting avenues for future research, which we briefly sketch hereafter.

First, we expect that the knowledge of the dynamical quadrupoles will allow for an improved description of the interatomic force constants, thereby enabling a more accurate computation of the phonon band structures. This refined processing of the IFCs might be important for certain material classes, such as piezoelectrics, where the treatment of long-range electrostatics is crucial for reproducing the correct sound velocity [36]. On a different note, our theory might also prove itself very helpful in establishing higher-order multipolar generalizations [37] of the Berry-phase theory of polarization [38]. Indeed, our expressions for the dynamical quadrupole and flexoelectric tensors can be regarded as the linear variation of the "bulk quadrupolization" [39] with respect to a zone-center lattice distortion or uniform strain, respectively. There are intriguing parallels to the theory of multipolar magnetic orders $[40,41]$ as well, which will certainly stimulate further studies.

Second, the treatment of flexoelectric effects beyond the clamped-ion level should be relatively straightforward by following the same guidelines as we do here. Both the "mixed" and "lattice-mediated" contributions involve first or second derivatives of the polarization response to a phonon, or the force-constant matrix, just like the electronic contribution. These additional pieces involve similar formulas, only with a slightly different combination of the basic response functions (electric field, atomic displacement, or uniform strain). Thus, the calculation of the full flexoelectric tensor for an insulating crystal or nanostructure of arbitrary symmetry looks now well within reach. We expect that, once implemented, it will involve a computational effort that is comparable to the calculation of the piezoelectric tensor.

Third, the method can be easily adapted to compute other spatial dispersion effects, for example, the natural optical rotation tensor. (The latter can be written as the first gradient with respect to the wave vector of the dielectric tensor.) First-principles calculations of natural gyrotropy are starting to appear [42]. We expect that by bringing it within the scopes of DFPT, the formalism that we present here will greatly simplify the calculation of this interesting quantity as well. For example, it may help clarify the physical origin of the nonmagnetic circular dichroism that has recently been observed in $\mathrm{PbTiO}_{3} / \mathrm{SrTiO}_{3}$ superlattices $[43,44]$. In the context of ferroic materials, we also expect our method to facilitate the development of first-principlesbased continuum models [45] and effective Hamiltonians [46], where gradient-mediated couplings often play an important role.

More generally, our work reveals a profound connection between spatial dispersion and orbital magnetism that, in our opinion, deserves further attention. Whenever the polarization response to a perturbation is needed at first order in the wave vector $\mathbf{q}$, one of the contributions necessarily involves the wave-function response to a gradient of $\mathbf{A}$, and hence, to a uniform magnetic field. This contribution can be tentatively interpreted as a "gyrotropic" response and is present only in certain crystal classes; we are unable to discuss it here because of space limitations, but we regard it as yet another interesting topic for future studies. As a final note, the polarization response to an inhomogeneous perturbation (including strain [47]) has received considerable attention in the context of semiclassical wave-packet dynamics [48]. It is our hope that the theory we develop here will help bring the aforementioned results in closer contact with first-principles electronic structure theory and possibly help clarify the microscopic physical nature of the effects described in Ref. [47].

\section{ACKNOWLEDGMENTS}

We acknowledge the support of the Ministerio de Economia, Industria y Competitividad through Grants No. MAT2016-77100-C2-2-P and No. SEV-2015-0496 and of the Generalitat de Catalunya (Grant No. 2017 SGR1506). This project receives funding from the European Research Council under the European Union's Horizon 2020 research and innovation program (Grant Agreement No. 724529). Part of the calculations are performed at the Supercomputing Center of Galicia.

\section{APPENDIX A: RESPONSE TO THE ELECTROMAGNETIC VECTOR POTENTIAL IN THE LONG-WAVELENGTH LIMIT}

The functions $\left|u_{m \mathbf{k}, \gamma}^{A_{\alpha}}\right\rangle$ are the $\mathbf{q}$ derivatives of the functions $\left|u_{m \mathbf{k}, \mathbf{q}}^{A_{\alpha}}\right\rangle$, which are defined as the solutions of the Sternheimer equation, Eq. (30). In the following, we proceed to explicitly demonstrate Eq. (32) by first reviewing the coupling of a generic Hamiltonian to a vector potential field and subsequently by performing the formal expansion of Eq. (30) to first order in $\mathbf{q}$.

\section{Coupling to a vector potential field}

The coupling of a generic Hamiltonian to a vector potential field can be written as $[28,49]$

$$
\hat{H}\left(\mathbf{r}, \mathbf{r}^{\prime}\right)=\hat{H}^{(0)}\left(\mathbf{r}, \mathbf{r}^{\prime}\right) e^{i \mathcal{Q} \int_{\mathbf{r}^{\prime}}^{\mathbf{r}} \mathbf{A} \cdot d \ell},
$$

where the line integral is assumed to be taken along the straight path connecting $\mathbf{r}^{\prime}$ to $\mathbf{r}, \mathcal{Q}$ is the particle charge ( $\mathcal{Q}=-e$ for electrons), and we use atomic units; i.e., we set $\hbar=c=1$. The linear expansion of the above expression in powers of the vector potential components to first order yields 


$$
\hat{H}\left(\mathbf{r}, \mathbf{r}^{\prime}\right) \simeq \hat{H}^{(0)}\left(\mathbf{r}, \mathbf{r}^{\prime}\right)+i \mathcal{Q} \hat{H}^{(0)}\left(\mathbf{r}, \mathbf{r}^{\prime}\right) \int_{\mathbf{r}^{\prime}}^{\mathbf{r}} \mathbf{A} \cdot d \ell
$$

We consider a monochromatic A field written as a real constant times a complex phase of wave vector $\mathbf{q}$,

$$
A_{\alpha}(\mathbf{r})=\lambda_{\alpha} e^{i \mathbf{q} \cdot r}
$$

After taking the line integral, one obtains a closed expression for the first-order Hamiltonian in a coordinate representation,

$$
\hat{H}_{\mathbf{q}}^{A_{\alpha}}\left(\mathbf{r}, \mathbf{r}^{\prime}\right)=i \mathcal{Q} \hat{H}^{(0)}\left(\mathbf{r}, \mathbf{r}^{\prime}\right)\left(r_{\alpha}-r_{\alpha}^{\prime}\right) \frac{e^{i \mathbf{q} \cdot \mathbf{r}}-e^{i \mathbf{q} \cdot \mathbf{r}^{\prime}}}{i \mathbf{q} \cdot\left(\mathbf{r}-\mathbf{r}^{\prime}\right)}
$$

Note that the first-order Hamiltonian is related to the current-density operator as

$$
\hat{J}_{\alpha}(-\mathbf{q})=-\hat{H}_{\mathbf{q}}^{A_{\alpha}}
$$

which stems from the thermodynamic relationship $\mathbf{J}(\mathbf{r})=-\delta E / \delta \mathbf{A}(\mathbf{r})$.

In the long-wavelength context of this work, it is useful to expand the fraction on the right-hand side in powers of $\mathbf{q}$ and to move the incommensurate phase factor to the left,

$$
\frac{e^{i \mathbf{q} \cdot \mathbf{r}}-e^{i \mathbf{q} \cdot \mathbf{r}^{\prime}}}{i \mathbf{q} \cdot\left(\mathbf{r}-\mathbf{r}^{\prime}\right)}=e^{i \mathbf{q} \cdot \mathbf{r}} \sum_{n=0}^{\infty} \frac{(-i)^{n}}{(n+1) !}\left[\mathbf{q} \cdot\left(\mathbf{r}-\mathbf{r}^{\prime}\right)\right]^{n}
$$

The above expansion clarifies that the fraction simplifies to unity in the $\mathbf{q}=0$ limit, where the first-order Hamiltonian reads as

$$
\hat{H}_{\mathbf{q}=0}^{A_{\alpha}}=-i \mathcal{Q}\left[\hat{H}^{(0)}, r_{\alpha}\right]
$$

This provides a first sanity check of the present formalism: In the $\mathbf{q}=0$ limit, the current-density operator as defined in Eq. (A5) correctly reduces to the velocity operator times the particle charge,

$$
\hat{J}_{\alpha}=\mathcal{Q} \hat{v}_{\alpha}, \quad \hat{v}_{\alpha}=i\left[\hat{H}^{(0)}, r_{\alpha}\right] .
$$

To make further progress towards a practical formalism, it is useful to consider the cell-periodic part of the firstorder Hamiltonian in momentum space,

$$
\hat{H}_{\mathbf{k}, \mathbf{q}}^{A_{\alpha}}\left(\mathbf{r}, \mathbf{r}^{\prime}\right)=e^{-i(\mathbf{k}+\mathbf{q}) \cdot \mathbf{r}} \hat{H}_{\mathbf{q}}^{A_{\alpha}}\left(\mathbf{r}, \mathbf{r}^{\prime}\right) e^{i \mathbf{k} \cdot \mathbf{r}^{\prime}}
$$

This is a self-adjoint operator at any $\mathbf{q}$, and can be conveniently written as

$$
\hat{H}_{\mathbf{k}, \mathbf{q}}^{A_{\alpha}}=-\mathcal{Q} \sum_{n=0}^{\infty}\left[\sum_{\beta_{1}, \ldots, \beta_{n}} \frac{q_{\beta_{1}}, \ldots, q_{\beta_{n}}}{(n+1) !} \hat{H}_{\alpha \beta_{1}, \ldots, \beta_{n}}\right],
$$

where the individual terms in the summation stem from the k expansion of the unperturbed Hamiltonian,

$$
\hat{H}_{\alpha \beta_{1}, \ldots, \beta_{n}}=\frac{\partial^{n+1} \hat{H}_{\mathbf{k}}^{(0)}}{\partial k_{\alpha} \partial k_{\beta_{1}}, \ldots, \partial k_{\beta_{n}}} .
$$

Equation (A10) is the first-order perturbation associated with a modulated vector potential field, which appears in Eq. (30).

\section{Long-wave expansion}

By deriving both sides of the Sternheimer equation (30), with respect to $q_{\gamma}$, one obtains

$$
\left(\hat{H}_{\mathbf{k}}^{(0)}+a \hat{P}_{\mathbf{k}}-\epsilon_{n \mathbf{k}}^{(0)}\right)\left|u_{n \mathbf{k}, \gamma}^{A_{\alpha}}\right\rangle=-\left(\partial_{\gamma} \hat{H}_{\mathbf{k}}^{(0)}+a \partial_{\gamma} \hat{P}_{\mathbf{k}}\right)\left|u_{n \mathbf{k}}^{A_{\alpha}}\right\rangle-\partial_{\gamma} \hat{Q}_{\mathbf{k}} \partial_{\alpha} \hat{H}_{\mathbf{k}}^{(0)}\left|u_{n \mathbf{k}}^{(0)}\right\rangle-\frac{1}{2} \hat{Q}_{\mathbf{k}} \partial_{\alpha \gamma}^{2} \hat{H}_{\mathbf{k}}^{(0)}\left|u_{n \mathbf{k}}^{(0)}\right\rangle
$$

[We drop the superscript "(0)" on the ground-state Hamiltonian operator from now on, to simplify the notation.] One can now use the zeroth-order result Eq. (31) to achieve the following expression (we also use $\partial_{\alpha} \hat{Q}_{\mathbf{k}}=-\partial_{\alpha} \hat{P}_{\mathbf{k}}$ whenever appropriate),

$$
\left(\hat{H}_{\mathbf{k}}+a \hat{P}_{\mathbf{k}}-\epsilon_{n \mathbf{k}}\right)\left|u_{n \mathbf{k}, \gamma}^{A_{\alpha}}\right\rangle=\left(\partial_{\gamma} \hat{H}_{\mathbf{k}} \partial_{\alpha} \hat{Q}_{\mathbf{k}}-a \partial_{\gamma} \hat{P}_{\mathbf{k}} \partial_{\alpha} \hat{P}_{\mathbf{k}}-\partial_{\gamma} \hat{Q}_{\mathbf{k}} \partial_{\alpha} \hat{H}_{\mathbf{k}}-\frac{1}{2} \hat{Q}_{\mathbf{k}} \partial_{\alpha} \partial_{\gamma} \hat{H}_{\mathbf{k}}\right)\left|u_{n \mathbf{k}}^{(0)}\right\rangle .
$$

It is convenient, at this point, to separately treat the contributions that are symmetric and antisymmetric under $\alpha \gamma$ exchange.

\section{a. Symmetric part}

We have

$$
\begin{aligned}
\left(\hat{H}_{\mathbf{k}}+a \hat{P}_{\mathbf{k}}-\epsilon_{n \mathbf{k}}\right)\left(\left|u_{n \mathbf{k}, \gamma}^{A_{\alpha}}\right\rangle+\left|u_{n \mathbf{k}, \alpha}^{A_{\gamma}}\right\rangle\right)= & \left(-a \partial_{\gamma} \hat{P}_{\mathbf{k}} \partial_{\alpha} \hat{P}_{\mathbf{k}}-a \partial_{\alpha} \hat{P}_{\mathbf{k}} \partial_{\gamma} \hat{P}_{\mathbf{k}}+\partial_{\gamma} \hat{H}_{\mathbf{k}} \partial_{\alpha} \hat{Q}_{\mathbf{k}}+\partial_{\alpha} \hat{H}_{\mathbf{k}} \partial_{\gamma} \hat{Q}_{\mathbf{k}}\right. \\
& \left.-\partial_{\gamma} \hat{Q}_{\mathbf{k}} \partial_{\alpha} \hat{H}_{\mathbf{k}}-\partial_{\alpha} \hat{Q}_{\mathbf{k}} \partial_{\gamma} \hat{H}_{\mathbf{k}}-\hat{Q}_{\mathbf{k}} \partial_{\alpha} \partial_{\gamma} \hat{H}_{\mathbf{k}}\right)\left|u_{n \mathbf{k}}^{(0)}\right\rangle .
\end{aligned}
$$


The large parentheses on the right-hand side contain a total of seven terms. The third to the seventh can be rewritten more compactly by observing that $\partial_{\alpha \gamma}^{2}\left[\hat{Q}_{\mathbf{k}}, \hat{H}_{\mathbf{k}}\right]=0$, leading to

$$
\left(\hat{H}_{\mathbf{k}}+a \hat{P}_{\mathbf{k}}-\epsilon_{n \mathbf{k}}\right)\left(\left|u_{n \mathbf{k}, \gamma}^{A_{\alpha}}\right\rangle+\left|u_{n \mathbf{k}, \alpha}^{A_{\gamma}}\right\rangle\right)=\left(-a \partial_{\gamma} \hat{P}_{\mathbf{k}} \partial_{\alpha} \hat{P}_{\mathbf{k}}-a \partial_{\alpha} \hat{P}_{\mathbf{k}} \partial_{\gamma} \hat{P}_{\mathbf{k}}-\left[\hat{H}_{\mathbf{k}}, \partial_{\alpha \gamma}^{2} \hat{Q}_{\mathbf{k}}\right]\right)\left|u_{n \mathbf{k}}^{(0)}\right\rangle .
$$

The solution is given by

$$
\left|u_{n \mathbf{k}, \gamma}^{A_{\alpha}}\right\rangle+\left|u_{n \mathbf{k}, \alpha}^{A_{\gamma}}\right\rangle=-\partial_{\alpha \gamma}^{2} \hat{Q}_{\mathbf{k}}\left|u_{n \mathbf{k}}^{(0)}\right\rangle=\partial_{\alpha \gamma}^{2} \hat{P}_{\mathbf{k}}\left|u_{n \mathbf{k}}^{(0)}\right\rangle .
$$

To justify the above derivation, observe that

$$
\partial_{\alpha \gamma}^{2}(P P)=\partial_{\alpha} P \partial_{\gamma} P+\partial_{\gamma} P \partial_{\alpha} P+\partial_{\alpha \gamma}^{2} P P+P \partial_{\alpha \gamma}^{2} P
$$

Using the idempotency of $P$, this immediately leads to

$$
P \partial_{\alpha \gamma}^{2} P P=-P\left(\partial_{\gamma} P \partial_{\alpha} P+\partial_{\alpha} P \partial_{\gamma} P\right) P .
$$

Note that the response is purely "geometric," i.e., given only in terms of the ground-state wave functions, despite the response containing both valence- and conduction-band components, as the operator $\partial_{\alpha \gamma}^{2} \hat{P}_{\mathbf{k}}$ generally has both inner and cross-gap matrix elements.

\section{b. Antisymmetric part}

The antisymmetric part can be written as follows:

$$
\begin{aligned}
\left(\hat{H}_{\mathbf{k}}+a \hat{P}_{\mathbf{k}}-\epsilon_{n \mathbf{k}}\right)\left(\left|u_{n \mathbf{k}, \gamma}^{A_{\alpha}}\right\rangle-\left|u_{n \mathbf{k}, \alpha}^{A_{\gamma}}\right\rangle\right)= & \left(-a \partial_{\gamma} \hat{P}_{\mathbf{k}} \partial_{\alpha} \hat{P}_{\mathbf{k}}+a \partial_{\alpha} \hat{P}_{\mathbf{k}} \partial_{\gamma} \hat{P}_{\mathbf{k}}+\partial_{\gamma} \hat{H}_{\mathbf{k}} \partial_{\alpha} \hat{Q}_{\mathbf{k}}\right. \\
& \left.-\partial_{\alpha} \hat{H}_{\mathbf{k}} \partial_{\gamma} \hat{Q}_{\mathbf{k}}-\partial_{\gamma} \hat{Q}_{\mathbf{k}} \partial_{\alpha} \hat{H}_{\mathbf{k}}+\partial_{\alpha} \hat{Q}_{\mathbf{k}} \partial_{\gamma} \hat{H}_{\mathbf{k}}\right)\left|u_{n \mathbf{k}}^{(0)}\right\rangle .
\end{aligned}
$$

This can be expressed more compactly by using (anti)commutators,

$$
\left(\hat{H}_{\mathbf{k}}+a \hat{P}_{\mathbf{k}}-\epsilon_{n \mathbf{k}}\right)\left(\left|u_{n \mathbf{k}, \gamma}^{A_{\alpha}}\right\rangle-\left|u_{n \mathbf{k}, \alpha}^{A_{\gamma}}\right\rangle\right)=\left(-a\left[\partial_{\gamma} \hat{P}_{\mathbf{k}}, \partial_{\alpha} \hat{P}_{\mathbf{k}}\right]-\left\{\partial_{\gamma} \hat{H}_{\mathbf{k}}, \partial_{\alpha} \hat{P}_{\mathbf{k}}\right\}+\left\{\partial_{\alpha} \hat{H}_{\mathbf{k}}, \partial_{\gamma} \hat{P}_{\mathbf{k}}\right\}\right)\left|u_{n \mathbf{k}}^{(0)}\right\rangle .
$$

To recast the above equation into a more transparent form, it is useful to work out the following expression,

$$
\begin{aligned}
{\left[H, \partial_{\gamma} P \partial_{\alpha} P-\partial_{\alpha} P \partial_{\gamma} P\right] } & =\left[H, \partial_{\gamma} P\right] \partial_{\alpha} P-\left[H, \partial_{\alpha} P\right] \partial_{\gamma} P-\partial_{\gamma} P\left[\partial_{\alpha} P, H\right]+\partial_{\alpha} P\left[\partial_{\gamma} P, H\right] \\
& =-\left[\partial_{\gamma} H, P\right] \partial_{\alpha} P+\left[\partial_{\alpha} H, P\right] \partial_{\gamma} P+\partial_{\gamma} P\left[P, \partial_{\alpha} H\right]-\partial_{\alpha} P\left[P, \partial_{\gamma} H\right] \\
& =P \partial_{\gamma} H \partial_{\alpha} P-P \partial_{\alpha} H \partial_{\gamma} P-P \partial_{\gamma} P \partial_{\alpha} H+P \partial_{\alpha} P \partial_{\gamma} H \\
& =P\left\{\partial_{\gamma} H, \partial_{\alpha} P\right\}-P\left\{\partial_{\alpha} H, \partial_{\gamma} P\right\}
\end{aligned}
$$

where the fact that the expression must be applied to a valence ket is used to go from the third to the fourth line. The Sternheimer equation can then be rewritten as

$$
\begin{aligned}
\left(\hat{H}_{\mathbf{k}}+a \hat{P}_{\mathbf{k}}-\epsilon_{n \mathbf{k}}\right)\left(\left|u_{n \mathbf{k}, \gamma}^{A_{\alpha}}\right\rangle-\left|u_{n \mathbf{k}, \alpha}^{A_{\gamma}}\right\rangle\right)= & -\left(\hat{H}_{\mathbf{k}}+a \hat{P}_{\mathbf{k}}-\epsilon_{n \mathbf{k}}\right)\left[\partial_{\gamma} \hat{P}_{\mathbf{k}}, \partial_{\alpha} \hat{P}_{\mathbf{k}}\right]\left|u_{n \mathbf{k}}^{(0)}\right\rangle \\
& -\hat{Q}_{\mathbf{k}}\left(\left\{\partial_{\gamma} \hat{H}_{\mathbf{k}}, \partial_{\alpha} \hat{P}_{\mathbf{k}}\right\}+\left\{\partial_{\alpha} \hat{H}_{\mathbf{k}}, \partial_{\gamma} \hat{P}_{\mathbf{k}}\right\}\right)\left|u_{n \mathbf{k}}^{(0)}\right\rangle,
\end{aligned}
$$

and finally,

$$
\left(\hat{H}_{\mathbf{k}}+a \hat{P}_{\mathbf{k}}-\epsilon_{n \mathbf{k}}\right)\left|u_{n \mathbf{k}, \gamma}^{\mathrm{CG}}\right\rangle=-\hat{Q}_{\mathbf{k}}\left(\left\{\partial_{\gamma} \hat{H}_{\mathbf{k}}, \partial_{\alpha} \hat{P}_{\mathbf{k}}\right\}-\left\{\partial_{\alpha} \hat{H}_{\mathbf{k}}, \partial_{\gamma} \hat{P}_{\mathbf{k}}\right\}\right)\left|u_{n \mathbf{k}}^{(0)}\right\rangle,
$$

where we define

$$
\left|u_{n \mathbf{k}, \gamma}^{\mathrm{CG}}\right\rangle=\left|u_{n \mathbf{k}, \gamma}^{A_{\alpha}}\right\rangle-\left|u_{n \mathbf{k}, \alpha}^{A_{\gamma}}\right\rangle+\left[\partial_{\gamma} \hat{P}_{\mathbf{k}}, \partial_{\alpha} \hat{P}_{\mathbf{k}}\right]\left|u_{n \mathbf{k}}^{(0)}\right\rangle .
$$

By combining Eqs. (A16) and (A21), we recover Eq. (32). 


\section{APPENDIX B: q DERIVATIVES OF FIRST-ORDER HAMILTONIANS}

\section{First q derivative of atomic displacement Hamiltonian}

The first-order Hamiltonian with respect to an atomic displacement consists of a local potential plus a nonlocal separable contribution,

$$
\begin{aligned}
V_{\mathbf{q}}^{\mathrm{loc}, \tau_{\kappa \beta}}(\mathbf{G})= & -i\left(G_{\beta}+q_{\beta}\right) \frac{1}{\Omega} e^{-i \mathbf{G} \cdot \boldsymbol{\tau}_{\kappa}} v_{\kappa}^{\mathrm{loc}}(G+q), \\
V_{\mathbf{k}, \mathbf{q}}^{\mathrm{sep}, \tau_{\kappa \beta}}\left(\mathbf{G}, \mathbf{G}^{\prime}\right)= & -i\left(G_{\beta}+q_{\beta}-G_{\beta}^{\prime}\right) \frac{1}{\Omega} \sum_{\mu} e^{-i\left(\mathbf{G}-\mathbf{G}^{\prime}\right) \cdot \boldsymbol{\tau}_{\kappa}} \\
& \times e_{\mu \kappa} \zeta_{\mu \kappa}(\mathbf{k}+\mathbf{q}+\mathbf{G}) \zeta_{\mu \kappa}^{*}\left(\mathbf{k}+\mathbf{G}^{\prime}\right) .
\end{aligned}
$$

Note that the above formulas slightly differ from the standard implementations of DFPT [22]. This is because in formulating them we use a different convention [13] for the sublattice-dependent phase factors. Such a difference is rooted in our assumption of a displacement pattern of the type $e^{i \mathbf{q} \mathbf{R}_{\mathrm{I}}}$ instead of the typical one $\left(e^{i \mathbf{q} \mathbf{R}_{\mathbf{1}}}\right)$. This choice leads to a much simpler and physically transparent treatment of the long-wave expansion. At $\mathbf{q}=0$, the present theory reduces to the standard treatment.

By differentiating the above formulas, we arrive at the following expressions for the first $\mathbf{q}$ gradients $($ at $\mathbf{q}=0$ ) of the perturbation, which are necessary for the dynamical quadrupole calculations,

$$
V_{\gamma}^{\mathrm{loc}, \tau_{\kappa \beta}}(\mathbf{G})=-i \frac{1}{\Omega} e^{-i \mathbf{G} \boldsymbol{\tau}_{\kappa}}\left(\delta_{\beta \gamma} v_{\kappa}^{\mathrm{loc}}(G)+\frac{G_{\beta} G_{\gamma}}{G} v_{\kappa}^{\mathrm{loc}}(G)^{\prime}\right)
$$

$$
\begin{aligned}
V_{\mathbf{k}, \gamma}^{\mathrm{sep}, \tau_{\kappa \beta}}\left(\mathbf{G}, \mathbf{G}^{\prime}\right) \\
=-i \frac{1}{\Omega} \sum_{\mu} e^{-i\left(\mathbf{G}-\mathbf{G}^{\prime}\right) \boldsymbol{\tau}_{\kappa}} e_{\mu \kappa}\left[\delta_{\beta \gamma} \zeta_{\mu \kappa}(\mathbf{k}+\mathbf{G}) \zeta_{\mu \kappa}^{*}\left(\mathbf{k}+\mathbf{G}^{\prime}\right)\right. \\
\left.\quad+\left(G_{\beta}-G_{\beta}^{\prime}\right) \zeta_{\mu \kappa, \gamma}(\mathbf{k}+\mathbf{G}) \zeta_{\mu \kappa}^{*}\left(\mathbf{k}+\mathbf{G}^{\prime}\right)\right],
\end{aligned}
$$

where $G=|\mathbf{G}|, \quad v_{\kappa}^{\text {loc }}(G)^{\prime}$ is the first derivative of the spherical atomic pseudopotential, and $\zeta_{\mu \kappa, \gamma}(\mathbf{k}+\mathbf{G})$ is the $\mathbf{q}$ derivative along the $\gamma$ direction of the separable nonlocal projector.

\section{Second q derivative of metric perturbation Hamiltonian}

The first-order Hamiltonian of the metric perturbation is [26]

$$
\hat{H}_{\mathbf{k}, \mathbf{q}}^{(\beta)}=\hat{T}_{\mathbf{k}, \mathbf{q}}^{(\beta)}+\hat{V}_{\mathbf{k}, \mathbf{q}}^{\mathrm{PSP},(\beta)}+\hat{V}_{\mathbf{q}}^{H 0,(\beta)}+\hat{V}_{\mathbf{q}}^{\mathrm{XC} 0,(\beta)}+\hat{V}_{\mathbf{q}}^{\mathrm{geom},(\beta)},
$$

where the terms on the right-hand side correspond to the kinetic $(\hat{T})$, pseudopotential (PSP), Hartree $(H 0)$, exchange-correlation (XC0), and geometric contributions to the external potential. The pseudopotential term, in turn, consists of a local plus a separable contribution,

$$
V_{\mathbf{k}, \mathbf{q}}^{\mathrm{PS},(\beta)}\left(\mathbf{G}, \mathbf{G}^{\prime}\right)=V_{\mathbf{q}}^{\mathrm{loc},(\beta)}\left(\mathbf{G}-\mathbf{G}^{\prime}\right)+V_{\mathbf{k}, \mathbf{q}}^{\mathrm{sep},(\beta)}\left(\mathbf{G}, \mathbf{G}^{\prime}\right)
$$

The explicit formulas for each of these terms are reported in Ref. [26]. In the following, we list the formulas for the second $\mathbf{q}$ gradients (at $\mathbf{q}=0$ ) of these contributions required in the calculation of the clamped-ion flexoelectric tensor [see, e.g., Eq. (73)].

The kinetic contribution is

$$
\begin{aligned}
T_{\mathbf{k}, \gamma \delta}^{(\beta)}\left(\mathbf{G}, \mathbf{G}^{\prime}\right)= & -i\left(\delta_{\gamma \delta}\left(k_{\beta}+G_{\beta}\right)+\delta_{\beta \gamma} \frac{1}{2}\left(k_{\delta}+G_{\delta}\right)\right. \\
& \left.+\delta_{\beta \delta} \frac{1}{2}\left(k_{\gamma}+G_{\gamma}\right)\right) \delta_{\mathbf{G G}^{\prime}}
\end{aligned}
$$

The local part of the pseudopotential is

$$
V_{\gamma \delta}^{\mathrm{loc},(\beta)}(\mathbf{G})=-i \frac{1}{\Omega} \sum_{\kappa} e^{-i \mathbf{G} \tau_{\kappa}}\left[\frac{v_{\kappa}^{\mathrm{loc}}(G)^{\prime}}{G}\left(\delta_{\beta \delta} G_{\gamma}+\delta_{\beta \gamma} G_{\delta}+\delta_{\gamma \delta} G_{\beta}-\frac{G_{\beta} G_{\delta} G_{\gamma}}{G^{2}}\right)+\frac{v_{\kappa}^{\mathrm{loc}}(G)^{\prime \prime}}{G^{2}} G_{\beta} G_{\delta} G_{\gamma}\right],
$$

with $v_{\kappa}^{\text {loc }}(G)^{\prime \prime}$ being the second derivative of the spherical atomic pseudopotential.

The separable part of the pseudopotential is

$$
\begin{aligned}
V_{\mathbf{k}, \gamma \delta}^{\mathrm{sep},(\beta)}\left(\mathbf{G}, \mathbf{G}^{\prime}\right)= & -\frac{i}{\Omega} \sum_{\mu \kappa} e_{\mu \kappa} e^{-i\left(\mathbf{G}-\mathbf{G}^{\prime}\right) \tau_{\kappa}}\left[\left(\frac{3}{2} \delta_{\beta \gamma} \zeta_{\mu \kappa, \delta}(\mathbf{k}+\mathbf{G})+\frac{3}{2} \delta_{\beta \delta} \zeta_{\mu \kappa, \gamma}(\mathbf{k}+\mathbf{G})+\left(k_{\beta}+G_{\beta}\right) \zeta_{\mu \kappa, \delta \gamma}(\mathbf{k}+\mathbf{G})\right) \zeta_{\mu \kappa}^{*}\left(\mathbf{k}+\mathbf{G}^{\prime}\right)\right. \\
& +\frac{1}{2} \delta_{\beta \delta} \zeta_{\mu \kappa}(\mathbf{k}+\mathbf{G}) \zeta_{\mu \kappa, \gamma}^{*}\left(\mathbf{k}+\mathbf{G}^{\prime}\right)+\zeta_{\mu \kappa, \delta}(\mathbf{k}+\mathbf{G}) \zeta_{\mu \kappa, \gamma}^{*}\left(\mathbf{k}+\mathbf{G}^{\prime}\right)\left(k_{\beta}+G_{\beta}^{\prime}\right)+\frac{1}{2} \delta_{\beta \gamma} \zeta_{\mu \kappa}(\mathbf{k}+\mathbf{G}) \zeta_{\mu \kappa, \delta}^{*}\left(\mathbf{k}+\mathbf{G}^{\prime}\right) \\
& \left.+\zeta_{\mu \kappa, \gamma}(\mathbf{k}+\mathbf{G}) \zeta_{\mu \kappa, \delta}^{*}\left(\mathbf{k}+\mathbf{G}^{\prime}\right)\left(k_{\beta}+G_{\beta}^{\prime}\right)+\zeta_{\mu \kappa}(\mathbf{k}+\mathbf{G}) \zeta_{\mu \kappa, \delta \gamma}^{*}\left(\mathbf{k}+\mathbf{G}^{\prime}\right)\left(k_{\beta}+G_{\beta}^{\prime}\right)\right]
\end{aligned}
$$

with $\zeta_{\mu \kappa, \gamma \delta}(\mathbf{k}+\mathbf{G})$ being the second $\mathbf{q}$ derivative along the $\gamma$ and $\delta$ directions of the separable nonlocal projector. 
The remaining terms [26] include the XC and geometric contributions that vanish at second order in $\mathbf{q}$ and a Hartree contribution whose second $\mathbf{q}$ gradient is

$$
\begin{aligned}
\hat{V}_{\delta \gamma}^{H 0,(\beta)}(\mathbf{G})= & -i 8 \pi \frac{n^{(0)}(\mathbf{G})}{G^{2}} \\
& \times\left(\frac{4 G_{\beta} G_{\delta} G_{\gamma}}{G^{4}}-\frac{\delta_{\beta \delta} G_{\gamma}+\delta_{\beta \gamma} G_{\delta}+\delta_{\delta \gamma} G_{\beta}}{G^{2}}\right),
\end{aligned}
$$

where $n^{(0)}(\mathbf{G})$ refers to the ground-state electron density.

It is useful at this point to perform a further rearrangement of $\hat{H}_{\mathbf{k}, \gamma \delta}^{(\beta)}$ by defining

$$
\hat{H}_{\mathbf{k}, \gamma}^{(\beta \delta)}=\hat{H}_{\mathbf{k}, \gamma \delta}^{(\beta)}+\hat{H}_{\mathbf{k}, \beta \gamma}^{(\delta)}-\hat{H}_{\mathbf{k}, \beta \delta}^{(\gamma)} .
$$

This allows us to write the flexoelectric tensor directly in type-II form as

$$
\mu_{\alpha \gamma, \beta \delta}^{\mathrm{II}}=\frac{2}{\Omega} E_{\gamma}^{\mathcal{E}_{\alpha}^{*}(\beta \delta)},
$$

where

$$
\begin{aligned}
E_{\gamma}^{\mathcal{E}_{\alpha}^{*}(\beta \delta)}= & s \int_{\mathrm{BZ}}\left[d^{3} k\right] \sum_{m} E_{m \mathbf{k}, \gamma}^{\mathcal{E}_{\alpha}^{*}(\beta \delta)} \\
& +\underbrace{\frac{i}{2} \int_{\Omega} \int K_{\gamma}\left(\mathbf{r}, \mathbf{r}^{\prime}\right) n^{\mathcal{E}_{\alpha}}(\mathbf{r}) n^{\eta_{\beta \delta}}\left(\mathbf{r}^{\prime}\right) d^{3} r d^{3} r^{\prime}}_{T_{\mathrm{elst}}}
\end{aligned}
$$

and

$$
\begin{aligned}
E_{m \mathbf{k}, \gamma}^{\mathcal{E}_{\alpha}^{*}(\beta \delta)}= & \underbrace{i\left\langle u_{m \mathbf{k}}^{\mathcal{E}_{\alpha}}\left|\partial_{\gamma} \hat{H}_{\mathbf{k}}^{(0)}\right| u_{m \mathbf{k}}^{\eta_{\beta \delta}}\right\rangle}_{T_{1}}+\underbrace{i\left\langle u_{m \mathbf{k}}^{\mathcal{E}_{\alpha}}\left|\partial_{\gamma} \hat{Q}_{\mathbf{k}} \hat{\mathcal{H}}_{\mathbf{k}}^{\eta_{\beta \delta}}\right| u_{m \mathbf{k}}^{(0)}\right\rangle}_{T_{2}} \\
& +\underbrace{i\left\langle u_{m \mathbf{k}}^{(0)}\left|\hat{V}^{\mathcal{E}_{\alpha}} \partial_{\gamma} \hat{Q}_{\mathbf{k}}\right| u_{m \mathbf{k}}^{\eta_{\beta \delta}}\right\rangle}_{T_{3}}+\underbrace{\frac{1}{2}\left\langle u_{m \mathbf{k}}^{\mathcal{E}_{\alpha}}\left|\hat{H}_{\mathbf{k}, \gamma}^{(\beta \delta)}\right| u_{m \mathbf{k}}^{(0)}\right\rangle}_{T_{4}} \\
& +\underbrace{i\left\langle i u_{m \mathbf{k}, \gamma}^{A_{\alpha}} \mid u_{m \mathbf{k}}^{\eta_{\beta \delta}}\right\rangle}_{T_{5}} .
\end{aligned}
$$

\section{APPENDIX C: TREATMENT OF THE} ELECTROSTATIC DIVERGENCE AT $\mathrm{G}=0$

The local potential diverges at $\mathbf{G}=0$ because of the Coulomb singularity [22]

$$
v_{\kappa}^{\operatorname{loc}}(q) \sim-\frac{4 \pi}{q^{2}} Z_{\kappa},
$$

where $Z_{\kappa}$ is the bare pseudopotential charge. This means that the $\mathbf{q}$ derivatives of the local potential contribution to the first-order Hamiltonians that we discuss in the previous sections must be calculated with some care regarding the $\mathbf{G}=0$ component. To see this, it is useful to rewrite Eq. (C1) as follows:

$$
v_{\kappa}^{\mathrm{loc}}(q)=\frac{F_{\kappa}(q)}{q^{2}},
$$

where we introduce the auxiliary function

$$
F_{\kappa}(q) \sim-4 \pi Z_{\kappa}+\frac{q^{2}}{2} F_{\kappa}^{\prime \prime} .
$$

Regarding the atomic displacement perturbation, the above definitions lead to the following small-q expansion of the local potential part at $\mathbf{G}=0$,

$$
V_{\mathbf{q}}^{\mathrm{loc}, \tau_{\kappa \beta}}(\mathbf{G}=0) \sim-\frac{i q_{\beta}}{\Omega}\left(-\frac{4 \pi Z_{\kappa}}{q^{2}}+\frac{F_{\kappa}^{\prime \prime}}{2}\right) .
$$

Because of the assumption of short-circuit electrical boundary conditions, we drop the divergent term. This leaves us with a constant multiplied by $q_{\beta}$, which vanishes in the $\mathbf{q} \rightarrow 0$ limit. The $\mathbf{q}$ derivative does not vanish,

$$
V_{\gamma}^{\mathrm{loc}, \tau_{\kappa \beta}}(\mathbf{G}=0) \rightarrow-\frac{i}{2 \Omega} F_{\kappa}^{\prime \prime} \delta_{\beta \gamma},
$$

and we should in principle take it into account in the calculation of the quadrupolar tensor. However, in Eq. (56) the operator $\hat{H}_{\mathbf{k} \gamma}^{\tau_{\kappa \beta}}$ appears only between a conduction-band bra and a valence-band ket. By orthogonality, the above constant contribution is irrelevant and can be safely discarded.

Regarding the metric perturbation, recall that it vanishes in the $\mathbf{q} \rightarrow 0$ limit, as the aforementioned divergence in the local potential contribution exactly cancels with an opposite divergence in the " $H 0$ " term [26]. Within the present notation conventions, one has

$$
\begin{aligned}
V_{\mathbf{q}}^{\mathrm{loc}+H 0,(\beta)}(\mathbf{G}=0) & =-\frac{i}{\Omega} \frac{q_{\beta}}{q^{2}}\left(\sum_{\kappa} F_{\kappa}(q)-4 \pi \Omega n^{(0)}(\mathbf{G}=0)\right) \\
& =-\frac{i}{2 \Omega} q_{\beta} \sum_{\kappa} F_{\kappa}^{\prime \prime}+O\left(q^{3}\right),
\end{aligned}
$$

where in the last line we take into account that $F_{\kappa}(q=$ $0)=4 \pi Z_{\kappa}$ [22], that $n^{(0)}(\mathbf{G}=0)=\left[\left(\sum_{\kappa} Z_{\kappa}\right) / \Omega\right]$, and that odd terms in the Taylor expansion of $F_{K}(q)$ vanish due to the spherical symmetry of the local atomic potentials. The first $\mathbf{q}$ derivative of the above yields a well-defined constant,

$$
V_{\gamma}^{\mathrm{loc}+H 0,(\beta)}(\mathbf{G}=0)=-\frac{i}{2 \Omega} \delta_{\beta \gamma} \sum_{\kappa} F_{\kappa}^{\prime \prime} .
$$


Recall that the first $\mathbf{q}$ derivative of the metric wave perturbation coincides (modulo a factor of $i$ ) with the uniform strain perturbation. Hence, we conclude that to calculate the clamped-ion flexoelectric tensor, the $\mathbf{G}=0$ component of the local potential of the first-order strain Hamiltonian has to be corrected as

$$
V^{\mathrm{loc}, \eta_{\beta \delta}}(\mathbf{G}=0)=V_{\delta}^{\mathrm{loc},(\beta)}(\mathbf{G}=0)=-\frac{i}{2 \Omega} \delta_{\beta \delta} \sum_{\kappa} F_{\kappa}(q)^{\prime \prime}
$$

This correction is important in the calculation of the flexoelectric tensor, since the uniform strain operator in Eq. (73) appears sandwiched between two unperturbed valence states.

[1] A. Malashevich and I. Souza, Band Theory of Spatial Dispersion in Magnetoelectrics, Phys. Rev. B 82, 245118 (2010).

[2] S. Prosandeev, A. Malashevich, Z. Gui, L. Louis, R. Walter, I. Souza, and L. Bellaiche, Natural Optical Activity and Its Control by Electric Field in Electrotoroidic Systems, Phys. Rev. B 87, 195111 (2013).

[3] D. L. Portigal and E. Burstein, Acoustical Activity and Other First-Order Spatial Dispersion Effects in Crystals, Phys. Rev. 170, 673 (1968).

[4] A. K. Tagantsev, Piezoelectricity and Flexoelectricity in Crystalline Dielectrics, Phys. Rev. B 34, 5883 (1986).

[5] P. Zubko, G. Catalan, and A. K. Tagantsev, Flexoelectric Effect in Solids, Annu. Rev. Mater. Res. 43, 387 (2013).

[6] M. Stengel and D. Vanderbilt, First-Principles Theory of Flexoelectricity, in Flexoelectricity in Solids From Theory to Applications, edited by A. K. Tagantsev and P. V. Yudin (World Scientific Publishing Co., Singapore, 2016), Chap. 2, pp. 31-110.

[7] U. K. Bhaskar, N. Banerjee, A. Abdollahi, Z. Wang, D. G. Schlom, G. Rijnders, and G. Catalan, A Flexoelectric Microelectromechanical System on Silicon, Nat. Nanotechnol. 11, 263 (2016).

[8] H. Lu, C.-W. Bark, D. E. de los Ojos, J. Alcala, C. B. Eom, G. Catalan, and A. Gruverman, Mechanical Writing of Ferroelectric Polarization, Science 336, 59 (2012).

[9] J. Narvaez, F. Vasquez-Sancho, and G. Catalan, Enhanced Flexoelectric-like Response in Oxide Semiconductors, Nature (London) 538, 219 (2016).

[10] M. Born and K. Huang, Dynamical Theory of Crystal Lattices (Oxford University Press, Oxford, 1954).

[11] X. Gonze and C. Lee, Dynamical Matrices, Born Effective Charges, Dielectric Permittivity Tensors, and Interatomic Force Constants from Density-Functional Perturbation Theory, Phys. Rev. B 55, 10355 (1997).

[12] S. Baroni, S. de Gironcoli, and A. Dal Corso, Phonons and Related Crystal Properties from Density-Functional Perturbation Theory, Rev. Mod. Phys. 73, 515 (2001).

[13] M. Stengel, Flexoelectricity from Density-Functional Perturbation Theory, Phys. Rev. B 88, 174106 (2013).
[14] R. M. Martin, Piezoelectricity, Phys. Rev. B 5, 1607 (1972).

[15] We discuss this specific example here, as flexoelectricity is a paradigmatic case of dispersion effect where the theoretical efforts have been most successful in the recent past. The following considerations, however, qualitatively hold for the entire class of physical properties that we mention in the previous paragraph.

[16] J. Hong and D. Vanderbilt, First-Principles Theory of Frozen-Ion Flexoelectricity, Phys. Rev. B 84, 180101(R) (2011).

[17] J. Hong and D. Vanderbilt, First-Principles Theory and Calculation of Flexoelectricity, Phys. Rev. B 88, 174107 (2013).

[18] M. Stengel, Microscopic Response to Inhomogeneous Deformations in Curvilinear Coordinates, Nat. Commun. 4, 2693 (2013).

[19] M. Stengel, Surface Control of Flexoelectricity, Phys. Rev. B 90, 201112(R) (2014).

[20] C. E. Dreyer, M. Stengel, and D. Vanderbilt, CurrentDensity Implementation for Calculating Flexoelectric Coefficients, Phys. Rev. B 98, 075153 (2018).

[21] X. Gonze, Adiabatic Density-Functional Perturbation Theory, Phys. Rev. A 52, 1096 (1995).

[22] X. Gonze, First-Principles Responses of Solids to Atomic Displacements and Homogeneous Electric Fields: Implementation of a Conjugate-Gradient Algorithm, Phys. Rev. B 55, 10337 (1997).

[23] The following expression is justified only in TR symmetric materials. For simplicity, we assume TR symmetry throughout this work.

[24] X. Gonze, Perturbation Expansion of Variational Principles at Arbitrary Order, Phys. Rev. A 52, 1086 (1995).

[25] F. Favot and A. Dal Corso, Phonon Dispersions: Performance of the Generalized Gradient Approximation, Phys. Rev. B 60, 11427 (1999).

[26] A. Schiaffino, C. E. Dreyer, D. Vanderbilt, and M. Stengel, Metric-Wave Approach to Flexoelectricity within DensityFunctional Perturbation Theory, Phys. Rev. B 99, 085107 (2019).

[27] M. Stengel, N. A. Spaldin, and D. Vanderbilt, Electric Displacement as the Fundamental Variable in ElectronicStructure Calculations, Nat. Phys. 5, 304 (2009).

[28] A. M. Essin, A. M. Turner, J. E. Moore, and D. Vanderbilt, Orbital Magnetoelectric Coupling in Band Insulators, Phys. Rev. B 81, 205104 (2010).

[29] X. Gonze et al., ABINIT: First-Principles Approach to Material and Nanosystem Properties, Comput. Phys. Commun. 180, 2582 (2009).

[30] J. P. Perdew and Y. Wang, Accurate and Simple Analytic Representation of the Electron-Gas Correlation Energy, Phys. Rev. B 45, 13244 (1992).

[31] M. Stengel, Unified Ab Initio Formulation of Flexoelectricity and Strain-Gradient Elasticity, Phys. Rev. B 93, 245107 (2016).

[32] D. Vanderbilt, Berry-Phase Theory of Proper Piezoelectric Response, J. Phys. Chem. Solids 61, 147 (2000).

[33] D. R. Hamann, X. Wu, K. M. Rabe, and D. Vanderbilt, Metric Tensor Formulation of Strain in Density-Functional Perturbation Theory, Phys. Rev. B 71, 035117 (2005). 
[34] G. Sàghi-Szabó, R. E. Cohen, and H. Krakauer, FirstPrinciples Study of Piezoelectricity in $\mathrm{PbTiO}_{3}$, Phys. Rev. Lett. 80, 4321 (1998).

[35] M. Stengel and D. Vanderbilt, Quantum Theory of Mechanical Deformations, Phys. Rev. B 98, 125133 (2018).

[36] X. Wu, D. Vanderbilt, and D. R. Hamann, Systematic Treatment of Displacements, Strains, and Electric Fields in Density-Functional Perturbation Theory, Phys. Rev. B 72, 035105 (2005).

[37] W. A. Benalcazar, B. Andrei Bernevig, and T. L. Hughes, Quantized Electric Multipole Insulators, Science 357, 61 (2017).

[38] R. D. King-Smith and D. Vanderbilt, Theory of Polarization of Crystalline Solids, Phys. Rev. B 47, R1651 (1993).

[39] W. A. Wheeler, L. K. Wagner, and T. L. Hughes, ManyBody Electric Multipole Operators in Extended Systems, arXiv:1812.06990.

[40] C. Ederer and N. A. Spaldin, Towards a Microscopic Theory of Toroidal Moments in Bulk Periodic Crystals, Phys. Rev. B 76, 214404 (2007).

[41] N. A. Spaldin, M. Fechner, E. Bousquet, A. Balatsky, and L. Nordström, Monopole-Based Formalism for the Diagonal Magnetoelectric Response, Phys. Rev. B 88, 094429 (2013).
[42] S. S. Tsirkin, P. Aguado Puente, and I. Souza, Gyrotropic Effects in Trigonal Tellurium Studied from First Principles, Phys. Rev. B 97, 035158 (2018).

[43] S. S. Tsirkin, P. A. Puente, and I. Souza, Gyrotropic Effects in Trigonal Tellurium Studied from First Principles, Phys. Rev. B 97 (2018).

[44] P. Shafer, P. García-Fernández, P. Aguado-Puente, A. R. Damodaran, A. K. Yadav, C. T. Nelson, S.-L. Hsu, J. C. Wojdeł, J. Íñiguez, L. W. Martin, E. Arenholz, J. Junquera, and R. Ramesh, Emergent Chirality in the Electric Polarization Texture of Titanate Superlattices, Proc. Natl. Acad. Sci. U.S.A. 115, 915 (2018).

[45] A. Schiaffino and M. Stengel, Macroscopic Polarization from Antiferrodistortive Cycloids in Ferroelastic $\mathrm{SrTiO}_{3}$, Phys. Rev. Lett. 119, 137601 (2017).

[46] W. Zhong, D. Vanderbilt, and K. M. Rabe, Phase Transitions in $\mathrm{BaTiO}_{3}$ from First Principles, Phys. Rev. Lett. 73, 1861 (1994).

[47] L. Dong and Q. Niu, Geometrodynamics of Electrons in a Crystal under Position and Time-Dependent Deformation, Phys. Rev. B 98, 115162 (2018).

[48] D. Xiao, J. Shi, D. P. Clougherty, and Q. Niu, Polarization and Adiabatic Pumping in Inhomogeneous Crystals, Phys. Rev. Lett. 102, 087602 (2009).

[49] F. Mauri and S. G. Louie, Magnetic Susceptibility of Insulators from First Principles, Phys. Rev. Lett. 76, 4246 (1996). 